\title{
Coleotterofauna acquatica e semiacquatica del bacino idrografico del fiume Cecina (Toscana)
}

\author{
(Coleoptera: Gyrinidae, Haliplidae, Noteridae, Dytiscidae, Helophoridae, Georissidae, \\ Hydrochidae, Hydrophilidae, Hydraenidae, Elmidae, Dryopidae, Limnichidae, \\ Heteroceridae, Chrysomelidae)
}

\begin{abstract}
Riassunto: Il presente contributo riguarda la coleotterofauna acquatica e semiacquatica dell'intero bacino idrografico (acque lotiche e lentiche) del fiume Cecina (Toscana). Vengono citati complessivamente 120 taxa (specie e sottospecie), suddivisi in 14 famiglie: Gyrinidae (6), Haliplidae (5), Noteridae (1), Dytiscidae (31), Helophoridae (8), Georissidae (2), Hydrochidae (2), Hydrophilidae (20), Hydraenidae (26), Elmidae (8), Dryopidae (5), Limnichidae (1), Heteroceridae (4), Chrysomelidae (1). L'elenco comprende sia i dati presenti in letteratura che quelli derivanti da materiale inedito.
\end{abstract}

Abstract: Aquatic and semiaquatic Beetles from the drainage basin of the Cecina river (Tuscany) (Coleoptera: Gyrinidae, Haliplidae, Noteridae, Dytiscidae, Helophoridae, Georissidae, Hydrochidae, Hydrophilidae, Hydraenidae, Elmidae, Dryopidae, Limnichidae, Heteroceridae, Chrysomelidae).

This contribution deals with the aquatic and semiaquatic beetles of the drainage basin (running and standing waters) of the Cecina river (Tuscany). 120 taxa belonging to 14 families are listed: Gyrinidae (6), Haliplidae (5), Noteridae (1), Dytiscidae (31), Helophoridae (8), Georissidae (2), Hydrochidae (2), Hydrophilidae (20), Hydraenidae (26), Elmidae (8), Dryopidae (5), Limnichidae (1), Heteroceridae (4), Chrysomelidae (1). This list includes both bibliographic and unpublished data.

Key words: Coleoptera, aquatic beetles, Cecina river, Tuscany.

\section{INTRODUZIONE}

Ad oggi sono noti due soli contributi inerenti l'entomocenosi dei coleotteri acquatici di un intero bacino idrografico in territorio italiano, ed entrambi riguardano la regione Toscana, più precisamente il fiume Sieve (Cianferoni et al., 2014) e il torrente Pesa (Rocchi \& Terzani, 2016).

Con il presente contributo, analogamente alle pubblicazioni sopra citate, si intende presentare l'elenco faunistico della coleotterofauna acquatica all'interno dell'intero bacino idrografico del fiume Cecina.

\section{AREA DI STUDIO}

Il bacino idrografico oggetto del presente contributo, caratterizzato da una superficie di circa $\mathrm{km}^{2}$ 765 , si sviluppa in ambiente prevalentemente collinare, degradante verso la costa del mare Tirreno; è attraversato dal fiume Cecina, lungo circa $\mathrm{km} 78$, dai suoi affluenti e subaffluenti; comprende territori ubicati in gran parte nella provincia di Pisa e, in numero inferiore, nelle province di Siena, Livorno e Grosseto (Regione Toscana, 2005).

Il fiume Cecina nasce da più rami nella zona delle Colline Metallifere dell'Antiappennino toscano e sfocia nel mare Tirreno in località Marina di Cecina (LI); i suoi affluenti principali sono sulla destra i torrenti Cortolla, Fosci, Le Botra, Lopia, Lupicaia, Zambra e sulla sinistra i torrenti Pavone, Possera, Sterza, Trossa. Tra i subaffluenti principali si menzionano il torrente Ritasso (affluente di destra del torrente Sterza, caratterizzato da una evidente integrità ecologica) e il torrente Sellate (affluente di destra del torrente Fosci).

Oltre ai suddetti biotopi di acque lotiche nell'area ne sono presenti anche alcuni di acque lentiche; citiamo i seguenti da noi visitati: nel comune di Montecatini Val di Cecina (PI) lo stagno presso il cimitero

\footnotetext{
"Saverio Rocchi, Fabio Terzani, Alessandro Mascagni, Museo di Storia Naturale dell’Università degli Studi di Firenze, Sezione di Zoologia "La Specola", via Romana 17, 50125 Firenze, Italy.

E-mail: rocchisaverio@gmail.com; libellula.ter@gmail.com; mascagni.ale@gmail.com
} 
di Gello e il laghetto del podere Sant'Alessandro presso Gello, nel comune di Monteverdi Marittimo (PI) presso Canneto lo stagno in località Le Strade e alcune pozze temporanee in località Pian di Bernino, nel comune di Volterra (PI) pozze temporanee nella zona dei pozzi salati presso Saline di Volterra

Nel territorio sono inoltre presenti le due Riserve naturali provinciali "Monterufoli-Caselli" e "Berignone" e il Sito di importanza regionale (SIR) "Fiume Cecina da Berignone a Ponteginori".

\section{MATERIALI E METODI}

Le nostre prime ricerche risalgono al 1989 e sono proseguite saltuariamente negli anni successivi per poi essere intensificate nel periodo 2016-2020. Sono stati visitati sia biotopi di acque lotiche che lentiche, facendo uso del classico retino da acqua e di colini con maglie di varie misure, mentre Georissidae, Limnichidae e Heteroceridae sono stati raccolti inondando le rive dei corsi d'acqua. Ci siamo deliberatamente limitati a campionamenti qualitativi e non quantitativi, così da arrecare il minor disturbo possibile alle biocenosi presenti nei biotopi investigati.

I campionamenti hanno riguardato specie legate biologicamente ed ecologicamente ad ambienti acquatici e semiacquatici, pertanto i taxa elencati rientrano fra quelli definiti da Jäch (1998) come "True Water Beetles" (Gyrinidae, Haliplidae, Noteridae, Dytiscidae, Helophoridae, Hydrochidae, Hydrophilidae, Hydraenidae, Elmidae, Dryopidae), "Shore Beetles" (Georissidae, Limnichidae, Heteroceridae), "Phytophilous Water Beetles" (Chrysomelidae).

Tutti gli esemplari sono stati da noi direttamente determinati; la lista delle specie e dei relativi reperti noti per il bacino idrografico del fiume Cecina è stata compilata tenendo conto:

- dei dati desunti dalla letteratura, molti dei quali citati in nostri precedenti lavori: Mascagni \& Calamandrei (1996); Mascagni et al. (1997); Rocchi et al. (1999, 2002); Mascagni (2005a, 2005b); Rocchi (2005a, 2005b); Rocchi \& Terzani (2018);

- del materiale inedito conservato nelle nostre collezioni, per la maggior parte proveniente da ricerche personali;

- del materiale inedito esaminato e conservato in altre collezioni.

Per l'ordine sistematico e per la nomenclatura dei taxa citati abbiamo seguito Hájek, 2017 (Noteri- dae), Hájek \& Fery, 2019 (Gyrinidae), Vondel, 2017, 2019 (Haliplidae), Nilsson \& Hájek, 2020 (Dytiscidae), Przewoźny, 2020 (Helophoridae, Georissidae, Hydrochidae, Hydrophilidae), Jäch \& Skale, 2015 (Hydraenidae), Jäch \& Kodada, 2016 (Elmidae), Kodada \& Jäch, 2016 (Dryopidae), Hernando \& Ribera, 2016 (Limnichidae), Mascagni, 2016 (Heteroceridae), Silfverberg, 2010 (Chrysomelidae Donaciinae).

Poiché nel corso del tempo numerose specie di coleotteri hanno subito dei cambiamenti sistematici e nomenclaturali è possibile che un taxon sia indicato nella bibliografia con altri nomi rispetto a quelli presenti nell'attuale lavoro, tuttavia, sulla base della letteratura sopra riportata, è possibile rintracciare i diversi nomi che si sono cronologicamente succeduti.

I corotipi fondamentali (Vigna Taglianti et al., 1993, 1999) attribuiti alle varie specie sono stati revisionati e aggiornati sulla base delle distribuzioni contenute nei lavori sopra menzionati a proposito dell'ordine seguito per la sistematica e la nomenclatura; in considerazione della loro rilevanza faunistica (Minelli et al., 2005) sono stati altresì evidenziati gli endemiti.

Ogni taxon specifico è quindi corredato dai relativi reperti editi e inediti, nonché dal corotipo fondamentale con rispettiva sigla (come in Stoch \& Vigna Taglianti, 2005).

Per ogni reperto dedotto dalla letteratura si riportano i dati della località e in parentesi la relativa fonte bibliografica; i dati faunistici inediti comprendono: la località, l'eventuale stazione, l'eventuale idronimo, la data di raccolta, la sigla del raccoglitore/i, il numero degli esemplari e in parentesi la sigla della collezione dove gli esemplari sono conservati.

Le località dei reperti sono elencate in ordine alfabetico e sono generalmente riferite a toponimi esistenti sull'Atlante stradale d'Italia in scala 1:200.000 (Touring Club Italiano, 2006).

\section{ABBREVIAZIONI}

Raccoglitori: $\mathrm{AM}=$ Alessandro Mascagni; FC $=$ Fabio Cianferoni; $\mathrm{FT}=$ Fabio Terzani $; \mathrm{LP}=$ Lucia Pizzocaro; NS = Nino Sanfilippo; PS = Pietro Cosimi; $\mathrm{RP}=$ Rossano Papi; $\mathrm{SC}=$ Simone Cianfanelli; $\mathrm{SF}=$ Silvio Failla; $\mathrm{SR}=$ Saverio Rocchi.

Collezioni: $\mathrm{CAM}=$ Alessandro Mascagni, Scandicci (Firenze); $\mathrm{CFC}=$ Fabio Cianferoni, Firenze; $\mathrm{CFP}=$ Ferdinando Maria Piccioli, conservata e incorporata nella collezione generale del reparto di Entomologia del Museo di Storia Naturale dell'Università 
degli Studi di Firenze, "La Specola", Zoologia; CFT $=$ Fabio Terzani, conservata e incorporata nella collezione generale del reparto di Entomologia del Museo di Storia Naturale dell'Università degli Studi di Firenze, "La Specola", Zoologia, Firenze; CLF $=$ Leonardo Forbicioni, Portoferraio (Livorno); CLP = Lucia Pizzocaro, Vezza d'Oglio (Brescia); CNS = Nino Sanfilippo, conservata nel Museo Civico di Storia Naturale "Giacomo Doria", Genova; CRP = Rossano Papi, Castelfranco di Sopra (Arezzo); CSR = Saverio Rocchi, conservata nel reparto di Entomologia del Museo di Storia Naturale dell'Università degli Studi di Firenze, "La Specola", Zoologia, Firenze; MZUF = collezione generale del reparto di Entomologia del Museo di Storia Naturale dell'Università degli Studi di Firenze, "La Specola", Zoologia, Firenze.

Altre abbreviazioni: es. $=$ esemplare/i; f. $=$ fiume; $\mathrm{m}$ = quota sul livello del mare; $\mathrm{t}$. = torrente; $\mathrm{GR}=$ provincia di Grosseto; $\mathrm{LI}=$ provincia di Livorno; $\mathrm{PI}=$ provincia di Pisa; $\mathrm{SI}=$ provincia di Siena.

\section{ELENCO FAUNISTICO}

\section{Gyrinidae}

Gyrinus (Gyrinus) caspius Ménétriés, 1832

RePERTI (8): Canneto (PI), fosso di Malentrata, m 140, 15.VI.2016, SR, 3 es. (CSR); Canneto (PI), t. Ritasso, m 100, 15.VI.2016, LP, 1 es. (CLP), SR, 2 es. (CSR); Canneto (PI), t. Ritasso, m 164, 29.V.2018, SR, 1 es. (CSR); Gerfalco (GR), f. Cecina (Targioni Tozzetti, 1879); Ponte di Cecina (SI), f. Cecina, m 259, 26.VII.2007, SR, 12 es. (CSR); Radicondoli (SI), Bagni delle Galleraie, f. Cecina, m 340 (Rocchi, 2005a); Riparbella (PI), t. Le Botra (Guerrini et al., 2008); Riparbella (PI), t. Le Botra presso Mulino del Frullino, m 66-70, 16.V.2017, SR, 3 es. (CSR).

COROTIPo: Centroasiatico-Europeo-Mediterraneo (CEM).

Gyrinus (Gyrinus) dejeani Brullé, 1832

REPERTI (2): Gerfalco (GR), f. Cecina (Targioni Tozzetti, 1879); Riparbella (PI), t. Le Botra (Guerrini et al., 2008).

COROTIPO: Mediterraneo (MED).

Gyrinus (Gyrinus) paykulli G. Ochs, 1927

REPERTI (2): f. Cecina (GR), (Targioni Tozzetti, 1879);
Montieri (GR), f. Cecina (Angelini, 1984).

Corotipo: Sibirico-Europeo (SIE).

Gyrinus (Gyrinus) substriatus Stephens, 1828

RePerti (4): Cecina (LI) (Rocchi, 2005a); Fiume Cecina (PI), senza data, senza raccoglitore, 1 es. (CFP); Ponte di Cecina (SI), f. Cecina, m 259, 26.VII.2007, FT, 4 es. (CFT); Radicondoli (SI), Bagni delle Galleraie, f. Cecina, m 340 (Rocchi, 2005a).

Corotipo: W-Paleartico (WPA).

\section{Gyrinus (Gyrinus) urinator Illiger, 1807}

RePerti (9): Canneto (PI), fosso di Malentrata, m 140, 15.VI.2016, SR, 2 es. (CSR); Canneto (PI), t. Ritasso, m 100, 15.VI.2016, SR, 2 es. (CSR); Canneto (PI), t. Ritasso, m 100, m 164, 29.V.2018, FT, 1 es. (CFT), SR, 1 es. (CSR); Canneto (PI), t. Sterza, m 110, 4.VIII.2005, SR, 2 es. (CSR); Ponte di Cecina (SI), f. Cecina, m 259, 26.VII.2007, SR, 4 es. (CSR); Ponteginori (PI), t. Trossa, m 49, 8.VI.2014, SR, 1 es. (CSR); Radicondoli (SI), Bagni delle Galleraie, f. Cecina, m 340 (Rocchi, 2005a); Riparbella (PI), t. Le Botra (Guerrini et al., 2008); Riparbella (PI), t. Le Botra, presso Mulino del Frullino, $\mathrm{m}$ 66-70, 16.V.2017, FT, 1 es. (CFT).

Corotipo: Europeo-Mediterraneo (EUM).

Orectochilus (Orectochilus) villosus villosus (O.F. Müller, 1776)

REPERTI (4): Radicondoli (SI), Bagni delle Galleraie, f. Cecina, m 340 (Rocchi, 2005a); Canneto (PI), pozza sulla strada per Pian di Bernino, m 112, 21.VI.2018, SR, 1 es. (CSR); Castelnuovo di Val di Cecina (PI), t. Pavone, m 350, 9.VII.1998, FT, 4 es. (CFT); Fiume Cecina (PI), 18.VII.2007, senza raccoglitore, 1 es. (MZUF).

COROTIPO: Paleartico (PAL).

\section{Haliplidae}

Haliplus (Haliplidius) obliquus (Fabricius, 1787)

RePERTI (1): Ponte di Cecina (SI), f. Cecina, m 259, 26.VII.2007, SR, 2 es. (CSR).

CoRotipo: Europeo-Mediterraneo (EUM).

Haliplus (Liaphlus) flavicollis Sturm, 1834

REPERTI (1): Saline di Volterra (PI), f. Cecina, m 70 (Rocchi, 2005a).

Corotipo: Centroasiatico-Europeo (CAE). 
Haliplus (Liaphlus) variegatus Sturm, 1834

RePERTI (2): Canneto (PI), Le Strade, stagno, m 175, 21.VI.2018, FT, 1 es. (CFT), SR, 2 es. (CSR)

Canneto (PI), pozza sulla strada per Pian di Bernino, m 112, 21.VI.2018, FT, 2 es. (CFT), SR, 3 es. (CSR). CoRotipo: Turanico-Europeo-Mediterraneo (TEM).

\section{Haliplus (Neohaliplus) lineatocollis (Marsham, 1802)}

RePERTI (5): Canneto (PI), t. Ritasso, m 174, 29.V.2018, SR, 1 es. (CSR); Ponte di Cecina (SI), f. Cecina, m 259, 26.VII.2007, SR, 8 es. (CSR); Riparbella (PI), Botro del Massone (Guerrini et al., 2008); Saline di Volterra (PI), f. Cecina, m 70 (Rocchi, 2005a); Saline di Volterra (PI), botro del Peruccio, $m$ 64, 17.IX.2020, AM, SR, FT, 2 es. (CSR).

Corotipo: W-Paleartico (WPA).

Peltodytes (Peltodytes) rotundatus (Aubé, 1836) RePerti (12): Canneto (PI), Le Strade, stagno, m 175, 21.VI.2018, FT, 1 es. (CFT); Canneto (PI), t. Ritasso, m 164, 29.V.2018, SR, 3 es. (CSR); Canneto (PI), t. Sterza, m 110, 4.VIII.2005, SR, 3 es. (CSR); Casino di Terra (PI), t. Sterza, m 41 (Rocchi, 2005a); Gello (PI), laghetto presso podere Sant'Alessandro, m 165, 16.V.2017, SR, 1 es. (CSR); Gello (PI), stagno presso il cimitero, m 143, 16.V.2017, FC, 2 es. (CFC); Pomarance (PI), f. Cecina, m 83, 17.IX.2020, AM, SR, FT, 2 es. (CSR); Pomarance (PI), Molino di Possera, t. Possera, m 180, 8.VI.2014, LP, 1 es. (CLP); Ponte di Cecina (SI), f. Cecina, m 259, 26.VII.2007, SR, 1 es. (CSR); Ponteginori (PI), t. Trossa, m 49, 8.VI.2014, SR, 1 es. (CSR); Riparbella (PI), t. Lopia presso podere Santa Margherita, m 6063, 16.V.2017, SR, 1 es. (CSR); Saline di Volterra (PI), f. Cecina, m 70 (Rocchi, 2005a).

Corotipo: Europeo-Mediterraneo (EUM).

\section{Noteridae}

Noterus clavicornis (De Geer, 1774)

REPERTI (4): Canneto (PI), Le Strade, stagno, m 175, 21.VI.2018, FT, 1 es. (CFT); Gello (PI), laghetto artificiale (Guerrini et al., 2008); Gello (PI), stagno presso il cimitero, m 143, 16.V.2017, FT, 3 es. (CFT), SR, 2 es. (CSR); Saline di Volterra (PI), f. Cecina, m 70 (Rocchi, 2005a).

Corotipo: Asiatico-Europeo (ASE).

\section{Dytiscidae}

Agabus (Gaurodytes) biguttatus (Olivier, 1795)

RePERTI (4): Castelnuovo di Val di Cecina (PI), t. Pavone, $\mathrm{m} \mathrm{330,27.VIII.2003,} \mathrm{FT,} 1$ es. (CFT); Fiume Cecina (PI) (Targioni Tozzetti, 1879); Riparbella (PI), Botro del Massone (Guerrini et al., 2008); Riparbella (PI), t. Lopia presso podere Santa Margherita, m 6063, 16.V.2017, SR, 2 es. (CSR).

COROTIPO: Paleartico (PAL).

Agabus (Gaurodytes) bipustulatus (Linnaeus, 1767) Reperti (7): Casino di Terra (PI), t. Sterza, m 41 (Rocchi, 2005a); Castelnuovo di Val di Cecina (PI), t. Pavone, m 330 (Rocchi, 2005a); Gello (PI), stagno presso il cimitero, m 143, 16.V.2017, FT, 2 es. (CFT); Ponte di Cecina (SI), f. Cecina, m 259, 26.VII.2007, SR, 1 es. (CSR); Radicondoli (SI), Bagni delle Galleraie, f. Cecina, m 340 (Rocchi, 2005a); Riparbella (PI), t. Le Botra (Guerrini et al., 2008); Saline di Volterra (PI), f. Cecina, m 70 (Rocchi, 2005).

Corotipo: Paleartico (PAL).

Agabus (Gaurodytes) brunneus (Fabricius, 1798)

Reperti (3): Canneto (PI), t. Ritasso, m 164, 29.V.2018, SR, 2 es. (CSR); Riparbella (PI), t. Lopia presso podere Santa Margherita, m 60-63, 16.V.2017, SR, 1 es. (CSR); Saline di Volterra (PI), botro del Peruccio, m 64, 17.IX.2020, AM, SR, FT, 1 es. (CSR). COROTIPO: Europeo-Mediterraneo (EUM).

Agabus (Gaurodytes) conspersus (Marsham, 1802) RePERTI (1): Cecina (LI) (Rocchi, 2005a).

Corotipo: Paleartico (PAL).

Agabus (Gaurodytes) didymus (Olivier, 1795)

REPERTI (4): Canneto (PI), t. Ritasso, m 164, 29.V.2018, SR, 1 es. (CSR); Ponte di Cecina (SI), f. Cecina, m 259, 26.VII.2007, SR, 1 es. (CSR); Riparbella (PI), Botro del Massone (Guerrini et al., 2008); Riparbella (PI), t. Le Botra (Guerrini et al., 2008). COROTIPO: Europeo-Mediterraneo (EUM).

Agabus (Gaurodytes) nebulosus (Forster, 1771) RePerTi (3): Fiume Cecina (PI) (Targioni Tozzetti, 1879); Radicondoli (SI), Bagni delle Galleraie, f. Cecina, m 340 (Rocchi, 2005a); Riparbella (PI), t. Le Botra presso Mulino del Frullino, m 66-70, 16.V.2017, SR, 1 es. (CSR). 
CoRotipo: Turanico-Europeo-Mediterraneo (TEM).

Rhantus suturalis (W.S. Macleay, 1825)

REPERTI (2): Canneto (PI), pozza sulla strada per Pian di Bernino, m 112, 21.VI.2018, FT, 1 es. (CFT), SR, 2 es. (CSR); Gello (PI), stagno presso il cimitero, $\mathrm{m}$ 143, 16.V.2017, FT, 2 es. (CFT).

Corotipo: Subcosmopolita (SCO).

\section{Meladema coriacea Laporte, 1835}

REPERTI (1): Riparbella (PI), t. Le Botra (Guerrini et al., 2008).

Corotipo: Mediterraneo (MED).

\section{Cybister (Cybister) lateralimarginalis lateralimargi-} nalis (De Geer, 1774)

REPERTI (1): Gello (PI), laghetto artificiale (Guerrini et al., 2008).

Corotipo: Centroasiatico-Europeo-Mediterraneo (CEM).

Dytiscus marginalis marginalis Linnaeus, 1758

RePerti (1): Castelnuovo di Val di Cecina (PI), t. Pavone, $\mathrm{m} 330$ (Rocchi, 2005a).

Corotipo: Asiatico-Europeo (ASE).

\section{Hydaticus (Prodaticus) leander (Rossi, 1790)}

REPERTI (1): Canneto (PI), pozza sulla strada per Pian di Bernino, m 112, 21.VI.2018, FT, 1 es. (CFT), SR, 2 es. (CSR).

COROTIPO: Afrotropicale-Mediterraneo (AFM).

\section{Bidessus minutissimus (Germar, 1824)}

REPERTI (15): Canneto (PI), pozza fangosa presso t. Ritasso, m 174, 29.V.2018, SR, 1 es. (CSR); Canneto (PI), t. Ritasso, m 100, 15.VI.2016, SR, 1 es. (CSR); Canneto (PI), t. Sterza, m 110 (Rocchi, 2005a); Canneto (PI), t. Sterza, m 92, 29.V.2018, FT, 3 es. (CFT), SR, 3 es. (CSR); Casino di Terra (PI), t. Sterza, m 41 (Rocchi, 2005a); Cecina (LI), f. Cecina (Fery, 1992); Pomarance (PI), confluenza t. Sterza con botro del Risecco (Guerrini et al., 2008); Pomarance (PI), f. Cecina, m 83, 17.IX.2020, AM, SR, FT, 2 es. (CFT), 1 es. (CSR); Pomarance (PI), f. Cecina, $m$ 87, 4.VIII.2005, SR, 2 es. (CSR); Ponte di Cecina (SI), f. Cecina, m 259, 26.VII.2007, SR, 3 es. (CSR); Ponteginori (PI) (Fery, 1992); Ponteginori (PI), t. Trossa, m 49, 8.VI.2014, SR, 3 es. (CSR); Radicondoli (SI), Bagni delle Galleraie, f. Cecina, m 340 (Rocchi, 2005a); Riparbella (PI), t. Lopia presso podere Santa Margherita, m 60-63, 16.V.2017, FT, 1 es. (CFT), SR, 2 es. (CSR); Saline di Volterra (PI), f. Cecina (Fery, 1992).

COROTIPO: Europeo-Mediterraneo (EUM).

Hydroglyphus geminus (Fabricius, 1792)

RePerti (8): Canneto (PI), Le Strade, stagno, m 175, 21.VI.2018, FT, 2 es. (CFT); Canneto (PI), pozza fangosa presso t. Ritasso, m 174, 29.V.2018, SR, 1 es. (CSR); Canneto (PI), pozza sulla strada per Pian di Bernino, m 112, 21.VI.2018, FT, 3 es. (CFT), SR, 2 es. (CSR); Casino di Terra (PI), t. Sterza, m 41, 4.VIII.2005, SR, 2 es. (CSR); Cecina (LI), f. Cecina (Rocchi, 2005a); Ponte di Cecina (SI), f. Cecina, m 259, 26.VII.2007, SR, 2 es. (CSR); Ponteginori (PI), t. Trossa, m 49, 8.VI.2014, SR, 4 es. (CSR); Riparbella (PI), t. Lopia presso podere Santa Margherita, m 6063, 16.V.2017, FT, 1 es. (CFT).

Corotipo: Paleartico (PAL).

Yola bicarinata (Latreille, 1804)

RePerti (5): Canneto (PI), t. Sterza, m 110, 4.VIII.2005, SR, 3 es. (CSR); Cecina (LI), f. Cecina (Rocchi, 2005a); Pomarance (PI), f. Cecina, m 83, 17.IX.2020, AM, SR, FT, 1 es. (CSR); Ponteginori (PI), t. Trossa, m 49, 8.VI.2014, SR, 3 es. (CSR); Saline di Volterra (PI), f. Cecina, m 70 (Rocchi, 2005a). COROTIPO: Europeo-Mediterraneo (EUM).

Deronectes moestus inconspectus (Leprieur, 1876) REPERTI (9): Canneto (PI), t. Ritasso, m 100, 15.VI.2016, SR, 1 es. (CSR); Canneto (PI), t. Ritasso, m 164, 29.V.2018, SR, 2 es. (CSR); Canneto (PI), t. Sterza, m 110 (Rocchi, 2005a); Castelnuovo di Val di Cecina (PI), t. Pavone, m 330 (Rocchi, 2005a); Ponte di Cecina (SI), f. Cecina, m 259, 26.VII.2007, SR, 7 es. (CSR); Radicondoli (SI), Bagni delle Galleraie, $\mathrm{f}$. Cecina, m 340 (Rocchi, 2005a); Riparbella (PI), t. Le Botra (Guerrini et al., 2008); Riparbella (PI), t. Lopia presso podere Santa Margherita, m 60-63, 16.V.2017, FT, 1 es. (CFT), SR, 3 es. (CSR); Saline di Volterra (PI), botro del Peruccio, m 64, 17.IX.2020, AM, SR, FT, 1 es. (CSR).

Corotipo: Mediterraneo (MED).

Graptodytes flavipes (Olivier, 1795)

RePerti (1): Cecina (LI) (Rocchi, 2005a).

COROTIPO: Europeo-Mediterraneo (EUM). 
Graptodytes varius (Aubé, 1838)

RePerti (11): Canneto (PI), t. Ritasso, m 100, 15.VI.2016, SR, 2 es. (CSR); Canneto (PI), t. Sterza, m 110 (Rocchi, 2005a); Casino di Terra (PI), t. Sterza, m 41 (Rocchi, 2005a); Castelnuovo di Val di Cecina (PI), t. Pavone, m 330 (Rocchi, 2005a); Gello (PI), laghetto artificiale (Guerrini et al., 2008); Ponte di Cecina (SI), f. Cecina, m 259, 26.VII.2007, SR, 9 es. (CSR); Riparbella (PI), Botro del Massone (Guerrini et al., 2008); Riparbella (PI), t. Le Botra (Guerrini et al., 2008); Riparbella (PI), t. Lopia presso podere Santa Margherita, m 60-63, 16.V.2017, FT, 1 es. (CFT), SR, 2 es. (CSR); Saline di Volterra (PI), f. Cecina, $\mathrm{m} 70$ (Rocchi, 2005a); Saline di Volterra (PI), botro del Peruccio, m 64, 17.IX.2020, AM, SR, FT, 2 es. (CSR).

Corotipo: Mediterraneo (MED).

Graptodytes veterator veterator (A. Zimmermann, 1918)

RePerti (4): Cecina (LI), f. Cecina (Rocchi, 2005a); Gello (PI), laghetto artificiale (Guerrini et al., 2008); Ponte di Cecina (SI), f. Cecina, m 259, 26.VII.2007, SR, 3 es. (CSR); Saline di Volterra (PI), f. Cecina, m 70 (Rocchi, 2005a).

Corotipo: E-Mediterraneo (EME).

Hydroporus analis Aubé, 1838

RePerti (1): Ponte di Cecina (SI), f. Cecina, m 259, 26.VII.2007, SR, 1 es. (CSR).

Corotipo: W-Mediterraneo (WME).

\section{Hydroporus memnonius Nicolai, 1822}

REPERTI (4): Canneto (PI), Le Strade, stagno, m 175, 21.VI.2018, SR, 3 es. (CSR); Gello (PI), stagno presso il cimitero, m 143, 16.V.2017, SR, 1 es. (CSR); Pomarance (PI), t. Trossa, m 200 (Rocchi, 2005a); Riparbella (PI), t. Lopia presso podere Santa Margherita, m 60-63, 16.V.2017, FT, 1 es. (CFT), SR, 1 es. (CSR). CoRotipo: Europeo-Mediterraneo (EUM).

Hydroporus obsoletus Aubé, 1838

REPERTI (1): Radicondoli (SI), Bagni delle Galleraie, f. Cecina, m 340 (Rocchi, 2005a).

CoRotipo: Europeo-Mediterraneo (EUM).

Hydroporus pubescens (Gyllenhal, 1808)

REPERTI (3): Cecina (LI) (Rocchi, 2005a); Radicondoli (SI), Bagni delle Galleraie, f. Cecina, m 340 (Rocchi, 2005a); Riparbella (PI), t. Lopia presso podere Santa
Margherita, m 60-63, 16.V.2017, FT, 1 es. (CFT). COROTIPO: Europeo-Mediterraneo (EUM).

Hydroporus tessellatus (Drapiez, 1819)

RePERTI (9): Canneto (PI), pozze temporanee nei pressi del t. Ritasso, m 100, 15.VI.2016, SR, 1 es. (CSR); Miemo (PI) (Rocchi, 2005a); Monteguidi (SI), f. Cecina, m 200 (Rocchi, 2005a); Ponte di Cecina (SI), f. Cecina, m 259, 26.VII.2007, SR, 1 es. (CSR); Ponteginori (PI), t. Trossa, m 49, 8.VI.2014, SR, 1 es. (CSR); Radicondoli (SI), Bagni delle Galleraie, f. Cecina, m 340 (Rocchi, 2005a); Riparbella (PI), Botro del Massone (Guerrini et al., 2008); Riparbella (PI), t. Le Botra (Guerrini et al., 2008); Riparbella (PI), t. Lopia presso podere Santa Margherita, m 60-63, 16.V.2017, SR, 1 es. (CSR).

COROTIPO: Europeo-Mediterraneo (EUM).

Nebrioporus luctuosus (Aubé, 1838)

RePerTi (3): Canneto (PI), t. Sterza, m 110 (Rocchi, 2005a); Cecina (LI) (Targioni Tozzetti, 1879); Volterra (PI), f. Cecina, 1.X.1967, NS, 15 es. (CNS).

COROTIPO: S-Europeo (SEU).

\section{Porhydrus obliquesignatus (Bielz, 1852)}

Reperti (3): Canneto (PI), t. Sterza, m 110, 4.VIII.2005, SR, 1 es. (CSR); Gello (PI), laghetto artificiale (Guerrini et al., 2008); Saline di Volterra (PI), f. Cecina, m 70 (Rocchi, 2005a).

Corotipo: Turanico-Europeo (TUE).

\section{Scarodytes halensis (Fabricius, 1787)}

REPERTI (9): Canneto (PI), t. Sterza, m 110 (Rocchi, 2005a); Casino di Terra (PI), t. Sterza, m 41 (Rocchi, 2005a); Cecina (LI), f. Cecina (Rocchi, 2005a); Monteguidi (SI), f. Cecina, m 200 (Rocchi, 2005a); Pomarance (PI), f. Cecina, m 83, 17.IX.2020, AM, SR, FT, 5 es. (CFT), 2 es. (CSR); Ponte di Cecina (SI), f. Cecina, m 259, 26.VII.2007, SR, 15 es. (CSR); Ponteginori (PI), t. Trossa, m 49, 8.VI.2014, SR, 2 es. (CSR); Riparbella (PI), t. Lopia presso podere Santa Margherita, $\mathrm{m}$ 60-63, 16.V.2017, FT, 2 es. (CFT), SR, 3 es. (CSR); Saline di Volterra (PI), f. Cecina, m 70 (Rocchi, 2005a).

CoRotipo: Europeo-Mediterraneo (EUM).

Stictotarsus duodecimpustulatus (Fabricius, 1792) RePerTi (2): Ponte di Cecina (SI), f. Cecina, m 259, 26.VII.2007, SR, 6 es. (CSR); Ponteginori (PI), t. Trossa, m 49, 8.VI.2014, SR, 1 es. (CSR). 
COROTIPO: Europeo (EUR).

Hydrovatus cuspidatus (Kunze, 1818)

REPERTI (3): Gello (PI), laghetto artificiale (Guerrini et al., 2008); Gello (PI), stagno presso il cimitero, $\mathrm{m}$ 143, 16.V.2017, FT, 1 es. (CFT), SR, 2 es. (CSR); Saline di Volterra (PI), f. Cecina, m 70 (Rocchi, 2005a). CoRotipo: Turanico-Europeo-Mediterraneo (TEM).

\section{Laccophilus hyalinus (De Geer, 1774)}

RePERTI (12): Canneto (PI), fosso di Malentrata, $m$ 140, 15.VI.2016, SR, 2 es. (CSR); Canneto (PI), Riserva Naturale Monterufoli-Caselli, m 200, 19.VI.2016, RP, 2 es. (CRP); Canneto (PI), t. Ritasso, m 100, 15.VI.2016, LP, 1 es. (CLP); Canneto (PI), t. Ritasso, m 164, 29.V.2018, FT, 1 es. (CFT), SR, 1 es. (CSR); Canneto (PI), t. Ritasso, m 174, 29.V.2018, FT, 1 es. (CFT), SR, 1 es. (CSR); Canneto (PI), t. Sterza, m 110, 4.VIII.2005, SR, 3 es. (CSR); Casino di Terra (PI), t. Sterza, m 41 (Rocchi, 2005a); Cecina (LI), f. Cecina (Rocchi, 2005a); Ponte di Cecina (SI), f. Cecina, m 259, 26.VII.2007, SR, 2 es. (CSR); Radicondoli (SI), Bagni delle Galleraie, f. Cecina, m 340 (Rocchi, 2005a); Riparbella (PI), Botro del Massone (Guerrini et al., 2008); Saline di Volterra (PI), f. Cecina, m 70 (Rocchi, 2005a).

CoRotipo: Turanico-Europeo-Mediterraneo (TEM).

\section{Laccophilus minutus (Linnaeus, 1758)}

RePerti (3): Canneto (PI), pozza sulla strada per Pian di Bernino, m 112, 21.VI.2018, FT, 1 es. (CFT), SR, 2 es. (CSR); Gello (PI), laghetto artificiale (Guerrini et al., 2008); Saline di Volterra (PI), f. Cecina, m 70 (Rocchi, 2005a).

Corotipo: Paleartico (PAL).

\section{Laccophilus poecilus Klug, 1834}

REPERTI (1): Gello (PI), stagno presso il cimitero, $\mathrm{m}$ 143, 16.V.2017, SR, 2 es. (CSR).

Corotipo: Centroasiatico-Europeo-Mediterraneo (CEM).

\section{Helophoridae}

Helophorus (Helophorus) aquaticus (Linnaeus, 1758)

RePerti (1): Miemo (PI), 15.X.1977, SF, 2 es. (MZUF).

Corotipo: Sibirico-Europeo (SIE).
Helophorus (Helophorus) milleri Kuwert, 1886

REPERTI (7): Canneto (PI), pozza fangosa presso t. Ritasso, m 174, 29.V.2018, FT, 1 es. (CFT); Canneto (PI), pozza sulla strada per Pian di Bernino, m 112, 21.VI.2018, FT, 1 es. (CFT), SR, 1 es. (CSR); Casale Marittimo (PI), 12.V.2013, PS, 2 es. (CLF); Casole d'Elsa (SI), t. Sellate, m 230 (Rocchi et al., 2002); Gello (PI), laghetto presso podere Sant'Alessandro, m 165, 16.V.2017, FT, 1 es. (CFT), SR, 2 es. (CSR); Riparbella (PI), t. Le Botra presso Mulino del Frullino, m 66-70, 16.V.2017, FT, 1 es. (CFT); Riparbella (PI), t. Lopia presso podere Santa Margherita, m 60-63, 16.V.2017, FT, 1 es. (CFT), SR, 2 es. (CSR).

CoRotipo: S-Europeo (SEU).

Helophorus (Rhopalohelophorus) brevipalpis brevipalpis Bedel, 1881

REPERTI (17): Canneto (PI), fosso di Malentrata, m 140, 15.VI.2016, SR, 1 es. (CSR); Canneto (PI), pozza fangosa presso t. Ritasso, m 174, 29.V.2018, FT, 6 es. (CFT), SR, 4 es. (CSR); Canneto (PI), pozza sulla strada per Pian di Bernino, m 112, 21.VI.2018, FT, 6 es. (CFT), SR, 3 es. (CSR); Canneto (PI), pozze temporanee nei pressi del t. Ritasso, m 100, 15.VI.2016, SR, 1 es. (CSR); Canneto (PI), t. Ritasso, m 164, 29.V.2018, FT, 5 es. (CFT), SR, 3 es. (CSR); Canneto (PI), t. Ritasso, m 174, 29.V.2018, FT, 1 es. (CFT); Canneto (PI), t. Sterza, m 92, 29.V.2018, FT, 1 es. (CFT), SR, 1 es. (CSR); Casole d'Elsa (SI), t. Sellate, m 230 (Rocchi et al., 2002); Gello (PI), laghetto presso podere Sant'Alessandro, m 165, 16.V.2017, SR, 1 es. (CSR); Guardistallo (PI), Pian del Molino, f. Cecina, 10.V.1989, FT, 1 es. (CFT); Guardistallo (PI), t. Sterza, 10.V.1989, FT, 1 es. (CFT); Pomarance (PI), confluenza t. Sterza con botro del Risecco (Guerrini et al., 2008); Pomarance (PI), t. Trossa, m 200 (Rocchi et al., 2002); Ponteginori (PI), t. Trossa, $m$ 49, 8.VI.2014, SR, 3 es. (CSR); Radicondoli (SI), Bagni delle Galleraie, f. Cecina, m 340 (Rocchi, 2005b); Riparbella (PI), t. Le Botra presso Mulino del Frullino, m 66-70, 16.V.2017, SR, 1 es. (CSR); Riparbella (PI), t. Lopia presso podere Santa Margherita, m 60-63, 16.V.2017, FC, 1 es. (CFC), FT, 6 es. (CFT), SR, 3 es. (CSR). COROTIPO: Europeo (EUR).

Helophorus (Rhopalohelophorus) flavipes Fabricius, 1792

RePerti (2): Canneto (PI), pozza sulla strada per Pian di Bernino, m 112, 21.VI.2018, FT, 1 es. (CFT); Can- 
neto (PI), t. Ritasso, m 100, 15.VI.2016, SR, 1 es. (CSR).

COROTIPO: Europeo (EUR).

Helophorus (Rhopalohelophorus) griseus Herbst, 1793

RePERTI (4): Canneto (PI), pozza sulla strada per Pian di Bernino, m 112, 21.VI.2018, SR, 1 es. (CSR); Gello (PI), laghetto presso podere Sant'Alessandro, m 165, 16.V.2017, FT, 1 es. (CFT); Ponte di Cecina (SI), f. Cecina, m 259, 26.VII.2007, SR, 1 es. (CSR); Riparbella (PI), t. Lopia presso podere Santa Margherita, m 60-63, 16.V.2017, SR, 1 es. (CSR).

COROTIPO: Europeo (EUR).

\section{Helophorus (Rhopalohelophorus) montenegrinus} Kuwert, 1885

REPERTI (1): Riparbella (PI), t. Lopia presso podere Santa Margherita, m 60-63 (Rocchi \& Terzani, 2018). CoRotipo: S-Europeo (SEU).

\section{Helophorus (Rhopalohelophorus) obscurus Mulsant, 1844}

Reperti (17): Canneto (PI), fosso di Malentrata, m 140, 15.VI.2016, SR, 1 es. (CSR); Canneto (PI), pozza fangosa presso t. Ritasso, m 174, 29.V.2018, FT, 3 es. (CFT), SR, 1 es. (CSR); Canneto (PI), pozza sulla strada per Pian di Bernino, $m$ 112, 21.VI.2018, FT, 2 es. (CFT), SR, 1 es. (CSR); Canneto (PI), pozze temporanee nei pressi del t. Ritasso, m 100, 15.VI.2016, SR, 1 es. (CSR); Canneto (PI), t. Ritasso, m 100, 15.VI.2016, LP, 3 es. (CLP), SR, 2 es. (CSR); Canneto (PI), t. Ritasso, $m$ 164, 29.V.2018, FT, 2 es. (CFT); Casole d'Elsa (SI), t. Sellate, m 200, 20.VI.1993, FT, 3 es. (CFT); Castelnuovo di Val di Cecina (PI), t. Pavone, m 350, 9.VII.1998, FT, 1 es. (CFT); Gello (PI), laghetto presso podere Sant'Alessandro, m 165, 16.V.2017, FT, 4 es. (CFT), SR, 1 es. (CSR); Pomarance (PI), f. Cecina, m 80, 6.VII.1991, FT, 1 es. (CFT); Pomarance (PI), t. Trossa, m 200 (Rocchi et al., 2002); Ponteginori (PI), t. Trossa, m 49, 8.VI.2014, SR, 1 es. (CSR); Radicondoli (SI), Bagni delle Galleraie, f. Cecina, m 340 (Rocchi, 2005b); Riparbella (PI) (Angus \& Toledo, 2010); Riparbella (PI), Botro del Massone (Guerrini et al., 2008); Riparbella (PI), t. Le Botra presso Mulino del Frullino, m 66-70, 16.V.2017, FT, 4 es. (CFT), SR, 2 es. (CSR); Ripar- bella (PI), t. Lopia presso podere Santa Margherita, m 60-63, 16.V.2017, FC, 1 es. (CFC), FT, 4 es. (CFT), SR, 2 es. (CSR).

COROTIPO: Europeo (EUR).

Helophorus (Trichohelophorus) alternans Gené, 1836

REPERTI (3): Canneto (PI), pozza fangosa presso t. Ritasso, m 174, 29.V.2018, SR, 1 es. (CSR); Gello (PI), laghetto presso podere Sant'Alessandro, $\mathrm{m}$ 165, 16.V.2017, FT, 1 es. (CFT); Riparbella (PI), t. Le Botra presso Mulino del Frullino, m 66-70, 16.V.2017, FT, 1 es. (CFT).

COROTIPO: Europeo-Mediterraneo (EUM).

\section{Georissidae}

Georissus (Georissus) crenulatus (P. Rossi, 1794) REPERTI (1): Volterra (PI), confluenza del f. Cecina con rio del Gagno, m 70 (Mascagni, 2005a).

Corotipo: Sibirico-Europeo (SIE).

Georissus (Neogeorissus) costatus Laporte, 1840 REPERTI (1): Volterra (PI), confluenza del f. Cecina con rio del Gagno, m 70 (Mascagni, 2005a).

COROTIPO: Europeo-Mediterraneo (EUM).

\section{Hydrochidae}

\section{Hydrochus flavipennis Küster, 1852}

REPERTI (5): Canneto (PI), Le Strade, stagno, m 175, 21.VI.2018, SR, 1 es. (CSR); Casino di Terra (PI), f. Cecina (Rocchi et al., 2002); Casino di Terra (PI), t. Sterza, m 41 (Rocchi, 2005b); Casole d'Elsa (SI), t. Sellate, m 230 (Rocchi et al., 2002); Gello (PI), laghetto artificiale (Guerrini et al., 2008).

COROTIPO: Europeo-Mediterraneo (EUM).

Hydrochus grandicollis Kiesenwetter, 1870

Reperti (5): Casole d'Elsa (SI), t. Sellate, m 230 (Rocchi et al., 2002); Castelnuovo di Val di Cecina (PI), t. Pavone, m 330, 27.VIII.2003, SR, 2 es. (CSR); Gello (PI), stagno presso il cimitero, $\mathrm{m}$ 143, 16.V.2017, FT, 1 es. (CFT), SR, 3 es. (CSR); Radicondoli (SI), Bagni delle Galleraie, f. Cecina, m 340 (Rocchi et al., 2002); Radicondoli (SI), f. Cecina (Rocchi, 2005b).

COROTIPO: Mediterraneo (MED). 


\section{Hydrophilidae}

Anacaena bipustulata (Marsham, 1802)

REPERTI (11): Canneto (PI), pozza fangosa presso t. Ritasso, m 174, 29.V.2018, SR, 1 es. (CSR); Canneto (PI), t. Ritasso, m 100, 15.VI.2016 (Rocchi \& Terzani, 2018); Canneto (PI), t. Sterza, m 110, 4.VIII.2005, SR, 1 es. (CSR); Casole d'Elsa (SI), t. Sellate, m 200, 20.VI.1993, FT, 1 es. (CFT); Gello (PI), laghetto artificiale (Guerrini et al., 2008); Gello (PI), stagno presso il cimitero, m 143, 16.V.2017, SR, 2 es. (CSR); Guardistallo (PI), t. Sterza, m 41, 21.VIII.2001 (Rocchi \& Terzani, 2018); Ponte di Cecina (SI), f. Cecina, m 259, 26.VII.2007 (Rocchi \& Terzani, 2018); Radicondoli (SI), Bagni delle Galleraie, f. Cecina, m 340 (Rocchi, 2005b); Riparbella (PI), Botro del Massone (Guerrini et al., 2008); Riparbella (PI), t. Lopia presso podere Santa Margherita, m 60-63, 16.V.2017, FT, 3 es. (CFT), SR, 2 es. (CSR).

COROTIPO: Europeo-Mediterraneo (EUM).

\section{Anacaena globulus (Paykull, 1798)}

REPERTI (11): Canneto (PI), Le Strade, stagno, m 175, 21.VI.2018, FT, 1 es. (CFT); Canneto (PI), pozza sulla strada per Pian di Bernino, m 112, 21.VI.2018, FT, 1 es. (CFT); Canneto (PI), t. Ritasso, m 164, 29.V.2018, FT, 1 es. (CFT), SR, 1 es. (CSR); Canneto (PI), t. Sterza, m 110, 5.VIII.1996, FT, 6 es. (CFT); Canneto (PI), t. Sterza, m 92, 29.V.2018, FT, 1 es. (CFT), SR, 2 es. (CSR); Casino di Terra (PI), t. Sterza, m 41 (Rocchi, 2005b); Casole d'Elsa (SI), t. Sellate, m 200, 20.VI.1993, FT, 1 es. (CFT); Castelnuovo di Val di Cecina (PI), t. Pavone, m 350, 9.VII.1998, FT, 3 es. (CFT); Radicondoli (SI), Bagni delle Galleraie, f. Cecina, m 340 (Rocchi, 2005b); Riparbella (PI), Botro del Massone (Guerrini et al., 2008); Riparbella (PI), t. Le Botra (Guerrini et al., 2008).

CoROTIPO: Europeo-Mediterraneo (EUM).

\section{Anacaena limbata (Fabricius, 1792)}

REPERTI (12): Canneto (PI), pozza fangosa presso t. Ritasso, m 174, 29.V.2018, SR, 1 es. (CSR); Canneto (PI), t. Sterza, m 110, 5.VIII.1996, FT, 2 es. (CFT); Casole d'Elsa (SI), t. Sellate, m 200, 20.VI.1993, FT, 1 es. (CFT); Casole d'Elsa (SI), t. Sellate, $\mathrm{m} 230$ (Rocchi et al., 2002); Castelnuovo di Val di Cecina (PI), t. Pavone, m 350, 9.VII.1998, FT, 1 es. (CFT); Castelnuovo di Val di Cecina (PI), t. Pavone, m 330, 27.VIII.2003, FT, 2 es. (CFT); Gello (PI), stagno presso il cimitero, m 143,
16.V.2017, SR, 1 es. (CSR); Guardistallo (PI), t. Sterza, m 41, 21.VIII.2001, FT, 1 es. (CFT); Ponte di Cecina (SI), f. Cecina, m 259, 26.VII.2007, FT, 5 es. (CFT); Radicondoli (SI), Bagni delle Galleraie, f. Cecina, $\mathrm{m}$ 340, 3.VII.1998, AM, FT, 1 es. (CAM), 1 es. (CFT); Riparbella (PI), t. Le Botra presso Mulino del Frullino, m 66-70, 16.V.2017, FT, 1 es. (CFT), SR, 1 es. (CSR); Riparbella (PI), t. Lopia presso podere Santa Margherita, m 60-63, 16.V.2017, FT, 1 es. (CFT).

COROTIPO: Turanico-Europeo-Mediterraneo (TEM).

\section{Anacaena lutescens (Stephens, 1829)}

REPERTI (7): Canneto (PI), fosso di Malentrata, m 140, 15.VI.2016 (Rocchi \& Terzani, 2018); Canneto (PI), Le Strade, stagno, m 175, 21.VI.2018, FT, 3 es. (CFT), SR, 2 es. (CSR); Canneto (PI), pozze temporanee nei pressi del t. Ritasso, m 100, 15.VI.2016, SR, 2 es. (CSR); Canneto (PI), t. Ritasso, m 100, 15.VI.2016 (Rocchi \& Terzani, 2018); Casino di Terra (PI), t. Sterza, m 41 (Rocchi, 2005b); Ponte di Cecina (SI), f. Cecina, $m$ 259, 26.VII.2007 (Rocchi \& Terzani, 2018); Ponteginori (PI), t. Trossa, m 49, 8.VI.2014 (Rocchi \& Terzani, 2018).

CoRotipo: Turanico-Europeo-Mediterraneo (TEM).

Berosus (Berosus) affinis Brullé, 1835

REPERTI (1): Gello (PI), laghetto artificiale (Guerrini et al., 2008).

Corotipo: Mediterraneo (MED).

\section{Berosus (Berosus) hispanicus Küster, 1847}

REPERTI (6): Cecina (LI) (Schödl, 1993); Laiatico (PI), t. Sterza, m 80-140, 10.IX.2007, FT, 11 es. (CFT); Pomarance (PI), f. Cecina, m 83, 17.IX.2020, AM, SR, FT, 1 es. (CFT), 2 es. (CSR); Ponteginori (PI) (Schödl, 1993); Ponteginori (PI), t. Trossa, m 49, 8.VI.2014, SR, 1 es. (CSR); Saline di Volterra (PI), botro del Peruccio, m 64, 17.IX.2020, AM, SR, FT, 2 es. (CSR). COROTIPO: Mediterraneo (MED).

Enochrus (Lumetus) quadripunctatus (Herbst, 1797) REPERTI (1): Casole d'Elsa (SI), t. Sellate, m 230 (Rocchi et al., 2002).

Corotipo: Asiatico-Europeo (ASE).

Enochrus (Lumetus) testaceus (Fabricius, 1801) RePerTi (1): Gello (PI), stagno presso il cimitero, $\mathrm{m}$ 143, 16.V.2017, SR, 3 es. (CSR).

Corotipo: Sibirico-Europeo (SIE). 
Helochares (Helochares) lividus (Forster, 1771)

REPERTI (13): Canneto (PI), Le Strade, stagno, m 175, 21.VI.2018, FT, 1 es. (CFT), SR, 1 es. (CSR); Canneto (PI), pozza sulla strada per Pian di Bernino, m 112, 21.VI.2018, FT, 2 es. (CFT), SR, 2 es. (CSR); Canneto (PI), t. Ritasso, m 100, 15.VI.2016, LP, 1 es. (CLP); Canneto (PI), t. Sterza, m 110, 5.VIII.1996, FT, 1 es. (CFT); Casino di Terra (PI), t. Sterza, m 41 (Rocchi, 2005b); Casole d'Elsa (SI), t. Sellate, m 230 (Rocchi et al., 2002); Gello (PI), laghetto artificiale (Guerrini et al., 2008); Gello (PI), laghetto presso podere Sant'Alessandro, m 165, 16.V.2017, SR, 1 es. (CSR); Gello (PI), stagno presso il cimitero, $m$ 143, 16.V.2017, FC, 1 es. (CFC), SR, 1 es. (CSR); Pomarance (PI), f. Cecina, $\mathrm{m} 80$ (Rocchi et al., 2002); Pomarance (PI), f. Cecina, $\mathrm{m} \mathrm{87,} \mathrm{4.VIII.2005,} \mathrm{FT,} 1$ es. (CFT); Ponte di Cecina (SI), f. Cecina, m 259, 26.VII.2007, SR, 2 es. (CSR); Volterra (PI), f. Cecina, m 80 (Rocchi et al., 2002).

CoROTIPO: Europeo-Mediterraneo (EUM).

Limnohydrobius convexus Brullé, 1835

REPERTI (2): Canneto (PI), Le Strade, stagno, m 175, 21.VI.2018, SR, 1 es. (CSR); Radicondoli (SI), Bagni delle Galleraie, f. Cecina, m 340 (Rocchi \& Terzani, 2018).

CoRotipo: Mediterraneo (MED).

Hydrochara caraboides (Linnaeus, 1758)

RePERTI (1): Cecina (LI) (Targioni Tozzetti, 1879). COROTIPO: Sibirico-Europeo (SIE).

\section{Laccobius (Dimorpholaccobius) albescens Rotten-} berg, 1874

REPERTI (24): Bibbona (LI), t. Sterza presso fattoria Faltona, m 44, 2.VIII.1996, FT, 4 es. (CFT); Canneto (PI), pozza fangosa presso t. Ritasso, m 174, 29.V.2018, SR, 1 es. (CSR); Canneto (PI), pozza sulla strada per Pian di Bernino, m 112, 21.VI.2018, FT, 2 es. (CFT), SR, 2 es. (CSR); Canneto (PI), t. Ritasso, m 164, 29.V.2018, FT, 1 es. (CFT), SR, 1 es. (CSR); Canneto (PI), t. Sterza, m 110, 5.VIII.1996, FT, 2 es. (CFT); Canneto (PI), t. Sterza, m 92, 29.V.2018, FT, 1 es. (CFT); Casino di Terra (PI), f. Cecina, 8.VIII.1996, FT, 1 es. (CFT); Casino di Terra (PI), t. Sterza, m 41 (Rocchi, 2005b); Gello (PI), laghetto presso podere Sant'Alessandro, m 165, 16.V.2017, SR, 1 es. (CSR); Guardistallo (PI), Piano del Molino, f. Cecina (Rocchi et al., 2002); Guardistallo (PI), t.
Sterza presso podere Aizzi, 10.V.1989, FT, 1 es. (CFT); Laiatico (PI), t. Sterza, m 80-140, 10.IX.2007, FT, 1 es. (CFT); Monteguidi (SI), f. Cecina, m 200 (Rocchi, 2005b); Pomarance (PI), f. Cecina, m 80 (Rocchi et al., 2002); Pomarance (PI), f. Cecina, $\mathrm{m} 83$, 17.IX.2020, AM, SR, FT, 2 es. (CFT), 1 es. (CSR); Pomarance (PI), f. Cecina, m 87, 4.VIII.2005, FT, 1 es. (CFT); Ponte di Cecina (SI), f. Cecina, m 259, 26.VII.2007, SR, 2 es. (CSR); Ponteginori (PI), t. Trossa, 9.VIII.1996, FT, 3 es. (CFT); Ponteginori (PI), t. Trossa, m 49, 8.VI.2014, SR, 4 es. (CSR); Radicondoli (SI), Bagni delle Galleraie, f. Cecina, m 340, 3.VII.1998, AM, 1 es. (CAM); Riparbella (PI), t. Le Botra presso Mulino del Frullino, m 66-70, 16.V.2017, FT, 1 es. (CFT); Riparbella (PI), t. Lopia presso podere Santa Margherita, m 60-63, 16.V.2017, FT, 2 es. (CFT), SR, 3 es. (CSR); Saline di Volterra (PI), botro del Peruccio, m 64, 17.IX.2020, AM, SR, FT, 2 es. (CAM); Volterra (PI), f. Cecina, m 80 (Rocchi et al., 2002).

CoRotipo: S-Europeo (SEU).

Laccobius (Dimorpholaccobius) neapolitanus Rottenberg, 1874

REPERTI (20): Canneto (PI), fosso di Malentrata, $\mathrm{m}$ 140, 15.VI.2016, SR, 3 es. (CSR); Canneto (PI), pozza fangosa presso t. Ritasso, m 174, 29.V.2018, SR, 1 es. (CSR); Canneto (PI), t. Ritasso, m 100, 15.VI.2016, LP, 1 es. (CLP); SR, 5 es. (CSR); Canneto (PI), t. Ritasso, m 164, 29.V.2018, FT, 2 es. (CFT), SR, 1 es. (CSR); Canneto (PI), t. Sterza, m 110, 5.VIII.1996, FT, 1 es. (CFT); Canneto (PI), t. Sterza, m 92, 29.V.2018, FT, 1 es. (CFT), SR, 1 es. (CSR); Casino di Terra (PI), t. Sterza, m 41 (Rocchi, 2005b); Casole d'Elsa (SI), t. Sellate, m 200, 20.VI.1993, FT, 2 es. (CFT); Castelnuovo di Val di Cecina (PI), t. Pavone, m 330 (Rocchi, 2005b); Monteguidi (SI), f. Cecina, m 200 (Rocchi, 2005b); Pomarance (PI), f. Cecina, m 80 (Rocchi et al., 2002); Pomarance (PI), f. Cecina, $\mathrm{m} 83$, 17.IX.2020, AM, SR, FT, 2 es. (CSR); Pomarance (PI), t. Trossa, m 200 (Rocchi et al., 2002); Ponte di Cecina (SI), f. Cecina, m 259, 26.VII.2007, SR, 3 es. (CSR); Ponteginori (PI), t. Trossa, m 49, 8.VI.2014, SR, 2 es. (CSR); Radicondoli (SI), Bagni delle Galleraie, f. Cecina, m 340 (Rocchi, 2005b); Radicondoli (SI), f. Cecina, m 222, 18.VI.1998, FT, 3 es. (CFT); Riparbella (PI), Botro del Massone (Guerrini et al., 2008); Riparbella (PI), t. Le Botra presso Mulino del Frullino, m 66-70, 16.V.2017, SR, 1 es. (CSR); Ripar- 
bella (PI), t. Lopia presso podere Santa Margherita, $\mathrm{m}$ 60-63, 16.V.2017, SR, 1 es. (CSR).

COROTIPO: Europeo-Mediterraneo (EUM).

Laccobius (Dimorpholaccobius) obscuratus Rottenberg, 1874

RePerti (2): Castelnuovo di Val di Cecina (PI), t. Pavone, m 330 (Rocchi, 2005b); Ponte di Cecina (SI), f. Cecina, m 259 (Rocchi \& Terzani, 2018).

Corotipo: Turanico-Europeo (TUE).

Laccobius (Microlaccobius) alternus Motschulsky, 1855

REPERTI (7): Bibbona (LI), t. Sterza presso fattoria Faltona, m 44, 2.VIII.1996, FT, 7 es. (CFT); Casino di Terra (PI), t. Sterza, m 41 (Rocchi, 2005b); Casino di Terra (PI), t. Sterza, m 90, 8.VI.2014, FT, 1 es. (CFT); Guardistallo (PI), podere Aizzi, t. Sterza (Rocchi et al., 2002); Pomarance (PI), f. Cecina, m 80 (Rocchi et al., 2002); Pomarance (PI), f. Cecina, $\mathrm{m} \mathrm{87,}$ 4.VIII.2005, SR, 4 es. (CSR); Ponteginori (PI), t. Trossa, m 49, 8.VI.2014, LP, 3 es. (CLP).

COROTIPO: S-Europeo (SEU).

Laccobius (Microlaccobius) gracilis gracilis Motschulsky, 1855

RePerti (10): Canneto (PI), t. Ritasso, m 100, 15.VI.2016, LP, 1 es. (CLP), SR, 1 es. (CSR); Canneto (PI), t. Sterza, m 92, 29.V.2018, FT, 1 es. (CFT), SR, 1 es. (CSR); Casino di Terra (PI), t. Sterza, m 41, 4.VIII.2005, SR, 1 es. (CSR); Guardistallo (PI), podere Aizzi, t. Sterza (Rocchi et al., 2002); Pomarance (PI), f. Cecina, m 83, 17.IX.2020, AM, SR, FT, 2 es. (CFT), 2 es. (CSR); Ponteginori (PI), t. Trossa, m 49, 8.VI.2014, SR, 2 es. (CSR); Radicondoli (SI), Bagni delle Galleraie, f. Cecina, m 340 (Rocchi et al., 2002); Riparbella (PI), t. Le Botra presso Mulino del Frullino, m 66-70, 16.V.2017, SR, 1 es. (CSR); Riparbella (PI), t. Lopia presso podere Santa Margherita, m 60-63, 16.V.2017, SR, 1 es. (CSR); Volterra (PI), confluenza f. Cecina con rio del Gagno, 10.V.1989, AM, 1 es. (CAM); FT, 2 es. (CFT).

CoRotiPo: Turanico-Europeo-Mediterraneo (TEM).

Paracymus aeneus (Germar, 1824)

REPERTI (1): Saline di Volterra (PI), zona dei pozzi salati, piccole pozze, $\mathrm{m}$ 107, 17.IX.2020, AM, SR, FT, 1 es. (CAM), 5 es. (CSR), 3 es. (CFT).

CoRotipo: Cetroasiatico-Europeo-Mediterraneo (CEM).
Coelostoma (Coelostoma) hispanicum (Küster, 1848) Reperti (9): Canneto (PI), t. Ritasso, m 100, 15.VI.2016, LP, 1 es. (CLP); SR, 1 es. (CSR); Canneto (PI), t. Sterza, m 110 (Rocchi et al., 2002); Casino di Terra (PI), f. Cecina (Rocchi et al., 2002); Casino di Terra (PI), t. Sterza, $\mathrm{m} 41$ (Rocchi, 2005b); Pomarance (PI), f. Cecina, m 80 (Rocchi et al., 2002); Pomarance (PI), f. Cecina, m 83, 17.IX.2020, AM, SR, FT, 1 es. (CFT); Ponteginori (PI), t. Trossa, m 49, 8.VI.2014, SR, 1 es. (CSR); Riparbella (PI) (Rocchi, 2005b); Volterra (PI), f. Cecina, m 80 (Rocchi et al., 2002).

Corotipo: Mediterraneo (MED).

Coelostoma (Coelostoma) orbiculare (Fabricius, 1775)

RePERTI (2): Canneto (PI), Le Strade, stagno, m 175, 21.VI.2018, SR, 1 es. (CSR); Gello (PI), stagno presso il cimitero, m 143, 16.V.2017, FT, 4 es. (CFT), SR, 3 es. (CSR).

COROTIPO: Asiatico-Europeo (ASE).

Cercyon (Cercyon) granarius Erichson, 1837

REPERTI (1): Marina di Cecina (LI) (Chiesa, 1959).

Corotipo: Sibirico-Europeo (SIE).

\section{Hydraenidae}

Hydraena andreinii D'Orchymont, 1934

RePerti (3): Canneto (PI), t. Ritasso, m 100, 15.VI.2016, SR, 1 es. (CSR); Ponte di Cecina (SI), f. Cecina, m 259, 26.VII.2007, FT, 1 es. (CFT); Riparbella (PI), t. Le Botra presso Mulino del Frullino, $\mathrm{m}$ 66-70, 16.V.2017, SR, 1 es. (CSR).

Corotipo: S-Europeo (SEU). Endemita: Appenninico (APPE).

Hydraena devillei Ganglbauer, 1901

RePerTi (8): Canneto (PI), t. Ritasso, m 100, 15.VI.2016, SR, 1 es. (CSR); Castelnuovo di Val di Cecina (PI), t. Pavone, m 330, 27.VIII.2003, FT, 1 es. (CFT); Castelnuovo di Val di Cecina (PI), t. Pavone, m 350, 9.VII.1998, FT, 2 es. (CFT); Guardistallo (PI), t. Sterza, m 41, 21.VIII.2001, FT, 1 es. (CFT); Pomarance (PI), confluenza t. Sterza con botro del Risecco (Guerrini et al., 2008); Radicondoli (SI), Bagni delle Galleraie, f. Cecina, m 340, 3.VII.1998, FT, 4 es. (CFT); ibidem, 21.V.2003, 2 es., FT (CFT); Radicondoli (SI), f. Cecina, m 222, 18.VII.1998, FT, 1 es. (CFT). 
Corotipo: S-Europeo (SEU). Endemita: W-AlpinoAppenninico (ALWA).

\section{Hydraena heterogyna Bedel, 1898}

RePERTI (3): Casale Marittimo (PI), m 200 (Audisio \& De Biase, 2005); Riparbella (PI), t. Le Botra (Guerrini et al., 2008); Volterra (PI), f. Cecina, $\mathrm{m} 80$ (Rocchi et al., 1999).

Corotipo: S-Europeo (SEU). Endemita: Italiano (ITAL).

\section{Hydraena similis D'Orchymont, 1930}

RePERTI (5): Canneto (PI), t. Ritasso, m 100, 15.VI.2016, SR, 1 es. (CSR); Canneto (PI), t. Sterza, m 110 (Rocchi et al., 1999); Castelnuovo di Val di Cecina (PI), t. Pavone, m 330, 13.VIII.2004, FT, 4 es. (CFT); Castelnuovo di Val di Cecina (PI), t. Pavone, m 350, 9.VII.1998, FT, 2 es. (CFT); Radicondoli (SI), Bagni delle Galleraie, f. Cecina, m 340, 3.VII.1998, FT, 3 es. (CFT).

Corotipo: S-Europeo (SEU). Endemita: Italiano (ITAL).

\section{Hydraena solarii Pretner, 1930}

RePerti (1): Casale Marittimo (PI), rio di Casale Marittimo (PI) (Audisio \& De Biase, 2005).

Corotipo: S-Europeo (SEU). Endemita: Appenninico (APPE).

Hydraena spinipes Baudi di Selve, 1882

RePerti (4): Castelnuovo di Val di Cecina (PI), t. Pavone, m 330, 13.VIII.2004, FT, 1 es. (CFT); Ponte di Cecina (SI), f. Cecina, m 259, 26.VII.2007, FT, 3 es. (CFT), SR, 1 es. (CSR); Riparbella (PI), Botro del Massone (Guerrini et al., 2008); Riparbella (PI), t. Le Botra (Guerrini et al., 2008).

Corotipo: S-Europeo (SEU). Endemita: Italiano (ITAL).

\section{Hydraena subimpressa Rey, 1885}

REPERTI (21): Canneto (PI), pozze temporanee nei pressi del t. Ritasso, m 100, 15.VI.2016, SR, 2 es. (CSR); Canneto (PI), t. Ritasso, m 100, 15.VI.2016, SR, 1 es. (CSR); Canneto (PI), t. Ritasso, m 164, 29.V.2018, FT, 1 es. (CFT); Canneto (PI), t. Sterza, m 92, 29.V.2018, FT, 2 es. (CFT); Canneto (PI), t. Sterza, m 110, 5.VIII.1996, FT, 2 es. (CFT); Casino di Terra (PI), f. Cecina, m 40 (Rocchi et al., 1999); Casino di Terra (PI), t. Sterza, m 41, 21.VIII.2001, SR, 2 es.
(CSR); Casole d'Elsa (SI), t. Sellate, m 200 (Rocchi et al., 1999); Castelnuovo di Val di Cecina (PI), t. Pavone, $\mathrm{m}$ 330, 27.VIII.2003, FT, 1 es. (CFT), SR, 2 es. (CSR); Castelnuovo di Val di Cecina (PI), t. Pavone, m 350, 9.VII.1998, FT, 4 es. (CFT); Fiume Cecina (PI), 18.VII.2007, senza raccoglitore, 2 es. (MZUF); Guardistallo (PI), t. Sterza, m 41, 4.VIII.2005, FT, 6 es. (CFT); Pomarance (PI), f. Cecina, $m$ 83, 17.IX.2020, AM, SR, FT, 1 es. (CSR); Pomarance (PI), f. Cecina, m 87, 4.VIII.2005, FT, 1 es. (CFT); Ponte di Cecina (SI), f. Cecina, m 259, 26.VII.2007, FT, 3 es. (CFT), SR, 1 es. (CSR); Ponteginori (PI), t. Trossa, m 50 (Rocchi et al., 1999); Radicondoli (SI), Bagni delle Galleraie, f. Cecina, m 340, 3.VII.1998, FT, 3 es. (CFT); ibidem, 21.V.2003, FT, 1 es. (CFT); Riparbella (PI), Botro del Massone (Guerrini et al., 2008); Riparbella (PI), t. Le Botra (Guerrini et al., 2008); Riparbella (PI), t. Lopia presso podere Santa Margherita, m 60-63, 16.V.2017, FT, 4 es. (CFT), SR, 3 es. (CSR).

CoRotipo: S-Europeo (SEU).

Limnebius atomus (Duftschmid, 1805)

RePERTI (2): Laiatico (PI), t. Sterza, m 80-140, 10.IX.2007, FT, 3 es. (CFT); Pomarance (PI), f. Cecina, $\mathrm{m}$ 87, 4.VIII.2005, FT, 1 es. (CFT).

COROTIPO: Sibirico-Europeo (SIE).

Limnebius furcatus Baudi di Selve, 1872

RePERTI (8): Canneto (PI), Le Strade, stagno, m 175, 21.VI.2018, FT, 1 es. (CFT), SR, 2 es. (CSR); Canneto (PI), pozza sulla strada per Pian di Bernino, m 112, 21.VI.2018, FT, 2 es. (CFT), SR, 2 es. (CSR); Casino di Terra (PI), t. Sterza, m 41, 21.VIII.2001, FT, 4 es. (CFT), SR, 2 es. (CSR); ibidem, 4.VIII.2005, SR, 1 es. (CSR); Casino di Terra (PI), t. Sterza, m 90, 8.VI.2014, FT, 1 es. (CFT); Gello (PI), laghetto artificiale (Guerrini et al., 2008); Ponteginori (PI), t. Trossa, m 49, 8.VI.2014, SR, 2 es. (CSR); Volterra (PI), confluenza del f. Cecina con rio del Gagno, $\mathrm{m} 70$ (Mascagni \& Calamandrei, 1996).

COROTIPO: Europeo-Mediterraneo (EUM).

Limnebius mucronatus Baudi di Selve, 1872

REPERTI (2): Canneto (PI), t. Ritasso, m 164, 29.V.2018, FT, 1 es. (CFT); Radicondoli (SI), Bagni delle Galleraie, f. Cecina, m 340, 3.VII.1998, FT, 1 es. (CFT). Corotipo: S-Europeo (SEU). Endemita: Italiano (ITAL). 
Limnebius myrmidon Rey, 1883

REPERTI (2): Canneto (PI), t. Sterza, m 110 (Rocchi et al., 1999); Casino di Terra (PI), t. Sterza, m 41, 21.VIII.2001, SR, 2 es. (CSR).

COROTIPO: S-Europeo (SEU).

Limnebius nitiduloides Baudi di Selve, 1872

Reperti (9): Canneto (PI), t. Ritasso, m 100, 15.VI.2016, SR, 2 es. (CSR); Castelnuovo di Val di Cecina (PI), t. Pavone, m 330, 27.VIII.2003, FT, 2 es. (CFT), SR, 2 es. (CSR); Castelnuovo di Val di Cecina (PI), t. Pavone, m 350, 9.VII.1998, FT, 2 es. (CFT); Guardistallo (PI), t. Sterza, m 41, 4.VIII.2005, FT, 2 es. (CFT); Ponte di Cecina (SI), f. Cecina, m 259, 26.VII.2007, FT, 1 es. (CFT); Radicondoli (SI), Bagni delle Galleraie, f. Cecina, m 340, 3.VII.1998, FT, 6 es. (CFT); Riparbella (PI), Botro del Massone (Guerrini et al., 2008); Riparbella (PI), t. Le Botra (Guerrini et al., 2008); Riparbella (PI), t. Le Botra presso Mulino del Frullino, m 66-70, 16.V.2017, FT, 1 es. (CFT), SR, 1 es. (CSR).

Corotipo: S-Europeo (SEU). Endemita: W-AlpinoAppenninico (ALWA).

\section{Limnebius oblongus Rey, 1883}

RePERTI (1): Guardistallo (PI), t. Sterza, m 41, 21.VIII.2001, FT, 1 es. (CFT).

Corotipo: W-Mediterraneo (WME).

\section{Limnebius papposus Mulsant, 1844}

REPERTI (1): Volterra (PI), confluenza del f. Cecina con rio del Gagno, m 70 (Mascagni \& Calamandrei, 1996).

COROTIPO: Europeo (EUR).

\section{Limnebius perparvulus Rey, 1884}

REPERTI (10): Bibbona (LI), t. Sterza, presso fattoria di Faltona, m 44, 2.VIII.1996, FT, 4 es. (CFT); Canneto (PI), t. Ritasso, m 100, 15.VI.2016, SR, 2 es. (CSR); Canneto (PI), t. Ritasso, m 164, 29.V.2018, FT, 4 es. (CFT); Canneto (PI), t. Ritasso, m 174, 29.V.2018, FT, 2 es. (CFT); Canneto (PI), t. Sterza, m 92, 29.V.2018, FT, 2 es. (CFT); Casino di Terra (PI), f. Cecina, m 40 (Rocchi et al., 1999); Pomarance (PI), f. Cecina, m 83, 17.IX.2020, AM, SR, FT, 3 es. (CFT), 4 es. (CSR); Ponteginori (PI), t. Trossa, m 49, 8.VI.2014, SR, 1 es. (CSR); Riparbella (PI), t. Le Botra presso Mulino del Frullino, m 66-70, 16.V.2017, SR, 4 es. (CSR); Riparbella (PI), t. Lopia presso po- dere Santa Margherita, m 60-63, 16.V.2017, FT, 1 es. (CFT), SR, 2 es. (CSR).

Corotipo: Mediterraneo (MED).

Aulacochthebius exaratus (Mulsant, 1844)

REPERTI (4): Canneto (PI), pozza sulla strada per Pian di Bernino, m 112, 21.VI.2018, SR, 2 es. (CSR); Casino di Terra (PI), t. Sterza, m 41, 21.VIII.2001, FT, 1 es. (CFT), SR, 1 es. (CSR); Gello (PI), laghetto presso podere Sant'Alessandro, m 165, 16.V.2017, FT, 1 es. (CFT); Ponteginori (PI), t. Trossa, m 50 (Rocchi et al., 1999).

COROTIPO: Europeo-Mediterraneo (EUM).

Ochthebius (Asiobates) crenulatus Mulsant \& Rey, 1850

RePerti (21): Canneto (PI), fosso di Malentrata, m 140, 15.VI.2016, SR, 4 es. (CSR); Canneto (PI), pozza fangosa presso t. Ritasso, m 174, 29.V.2018, FT, 2 es. (CFT), SR, 2 es. (CSR); Canneto (PI), pozze temporanee nei pressi del t. Ritasso, m 100, 15.VI.2016, SR, 2 es. (CSR); Canneto (PI), t. Ritasso, m 100, 1.VI.2016, LP, 1 es. (CLP), SR, 3 es. (CSR); Canneto (PI), t. Ritasso, m 164, 29.V.2018, SR, 2 es. (CSR); Canneto (PI), t. Ritasso, m 174, 29.V.2018, SR. 1 (CSR); Canneto (PI), t. Sterza, m 92, 29.V.2018, FT, 1 es. (CFT), SR, 2 es. (CSR); Casole d'Elsa (SI), t. Sellate, m 200 (Rocchi et al., 1999); Castelnuovo di Val di Cecina (PI), t. Pavone, m 250 (Rocchi et al., 1999); Gello (PI), laghetto presso podere Sant'Alessandro, m 165, 16.V.2017, FT, 1 es. (CFT), SR, 2 es. (CSR); Guardistallo (PI), Pian di Molino, f. Cecina, m 40 (Rocchi et al., 1999); Monteguidi (SI), f. Cecina, m 200, 6.VI.2001, SR, 3 es. (CSR); Pomarance (PI), confluenza t. Sterza con botro del Risecco (Guerrini et al., 2008); Pomarance (PI), t. Trossa, m 200 (Rocchi et al., 1999); Ponte di Cecina (SI), f. Cecina, m 259, 26.VII.2007, FT, 1 es. (CFT), SR, 1 es. (CSR); Ponteginori (PI), t. Trossa, m 49, 8.VI.2014, SR, 3 es. (CSR); Radicondoli (SI), Bagni delle Galleraie, f. Cecina, m 340, 3.VII.1998, FT, 1 es. (CFT); Radicondoli (SI), f. Cecina, m 222, 18.VI.1998, FT, 3 es., (CFT); Riparbella (PI), t. Le Botra presso Mulino del Frullino, m 66-70, 16.V.2017, FT, 2 es. (CFT), SR, 3 es. (CSR); Riparbella (PI), t. Lopia presso podere Santa Margherita, m 60-63, 16.V.2017, FT, 4 es. (CFT), SR, 4 es. (CSR); Volterra (PI), f. Cecina, m 80 (Rocchi et al., 1999).

CoRotipo: S-Europeo (SEU). 
Ochthebius (Asiobates) dilatatus Stephens, 1829 REPERTI (3): Canneto (PI), pozza sulla strada per Pian di Bernino, m 112, 21.VI.2018, SR, 1 es. (CSR); Guardistallo (PI), Pian di Molino, f. Cecina, $\mathrm{m} 40$ (Rocchi et al., 1999); Pomarance (PI), confluenza t. Sterza con botro del Risecco (Guerrini et al., 2008).

CoRotipo: Europeo-Mediterraneo (EUM).

Ochthebius (Asiobates) gagliardii D'Orchymont, 1940

RePERTI (5): Canneto (PI), pozza fangosa presso t. Ritasso, m 174, 29.V.2018, FT, 1 es. (CFT), SR, 4 es. (CSR); Casino di Terra (PI), t. Sterza, m 90, 8.VI.2014, FT, 1 es. (CFT); Pomarance (PI), confluenza t. Sterza con botro del Risecco (Guerrini et al., 2008); Pomarance (PI), t. Trossa, m 200 (Rocchi et al., 1999); Ponteginori (PI), t. Trossa, m 49, 8.VI.2014, SR, 1 es. (CSR).

Corotipo: S-Europeo (SEU). Endemita: Italiano (ITAL).

Ochthebius (Enicocerus) halbherri (Reitter, 1890)

RePerti (1): Canneto (PI), t. Ritasso, m 100, 15.VI.2016, SR, 1 es. (CSR).

Corotipo: S-Europeo (SEU). Endemita: W-AlpinoAppenninico (ALWA).

Ochthebius (Ochthebius) difficilis Mulsant, 1844 RePERTI (3): Canneto (PI), pozza fangosa presso t. Ritasso, m 174, 29.V.2018, FT, 1 es. (CFT); Castelnuovo di Val di Cecina (PI), t. Pavone, $\mathrm{m} 250$ (Audisio \& De Biase, 2005); Pomarance (PI), f. Cecina, m 83, 17.IX.2020, AM, SR, FT, 4 es. (CFT), 3 es. (CSR).

Corotipo: Mediterraneo (MED).

Ochthebius (Ochthebius) foveolatus Germar, 1824 RePerti (4): Guardistallo (PI), Pian di Molino, f. Cecina, m 40 (Rocchi et al., 1999); Laiatico (PI), t. Sterza, 4.IV.1995, SC, 1 es. (MZUF); Pomarance (PI), f. Cecina, m 87, 4.VIII.2005, FT, 1 es. (CFT); Volterra (PI), f. Cecina, m 80 (Rocchi et al., 1999).

COROTIPO: S-Europeo (SEU).

Ochthebius (Ochthebius) lividipennis Peyron, 1858 REPERTI (1): Canneto (PI), pozza sulla strada per Pian di Bernino, m 112, 21.VI.2018, FT, 1 es. (CFT), SR, 1 es. (CSR).

COROTIPO: E-Mediterraneo (EME).
Ochthebius (Ochthebius) pusillus Stephens, 1835

RePERTI (1): Canneto (PI), pozza sulla strada per Pian di Bernino, m 112, 21.VI.2018, FT, 3 es. (CFT), SR, 4 es. (CSR).

COROTIPO: Centro-Europeo (CEU).

Ochthebius (Ochthebius) sidanus D'Orchymont, 1942

RePERTI (2): Montegemoli (PI), m 200 (Jäch, 1991); Pomarance (PI), f. Cecina, m 83, 17.IX.2020, AM, SR, FT, 1 es. (CSR).

CoRotipo: S-Europeo (SEU).

Ochthebius (Ochthebius) virgula Ferro, 1986

Reperti (4): Casino di Terra (PI), f. Cecina, m 40 (Rocchi et al., 1999); Laiatico (PI), t. Sterza, m 80140, 10.IX.2007, FT, 1 es. (CFT); Pomarance (PI), f. Cecina, m 83, 17.IX.2020, AM, SR, FT, 2 es. (CFT), 4 es. (CSR); Pomarance (PI), t. Trossa, m 200 (Audisio \& De Biase, 2005).

CoRotIPO: S-Europeo (SEU). Endemita: Italiano (ITAL).

\section{EImidae}

Elmis aenea (P.W.J. Müller, 1806)

Reperti (1): Canneto (PI), t. Ritasso, m 100, 15.VI.2016, FT, 1 es. (CFT).

COROTIPO: Europeo (EUR).

Elmis maugetii maugetii Latreille, 1802

RePerti (2): Castelnuovo di Val di Cecina (PI), t. Pavone, m 350 (Mascagni et al., 1997); Radicondoli (SI), Bagni delle Galleraie, f. Cecina, m 340, 3.VII.1998, FT, 1 es. (CFT).

CoRotipo: Turanico-Europeo-Mediterraneo (TEM).

Esolus berthelemyi Olmi, 1975

RePerti (1): Guardistallo (PI), t. Sterza, m 41, 21.VIII.2001, FT, 1 es. (CFT).

Corotipo: S-Europeo (SEU). Endemita: Italiano (ITAL).

Limnius intermedius Fairmaire, 1881

REPERTI (1): Radicondoli (SI), Bagni delle Galleraie, f. Cecina, m 340, 3.VII.1998, FT, 2 es. (CFT).

COROTIPO: Europeo-Mediterraneo (EUM).

Limnius opacus opacus P.W.J. Müller, 1806 REPERTI (1): Radicondoli (SI), Bagni delle Galleraie, 
f. Cecina, m 340, 21.V.2003, FT, 3 es. (CFT).

Corotipo: Europeo-Mediterraneo (EUM).

Riolus cupreus (P.W.J. Müller, 1806)

RePERTI (2): Canneto (PI), t. Ritasso, m 100, 15.VI.2016, SR, 1 es. (CSR); Pomarance (PI), f. Cecina, $\mathrm{m}$ 87, 4.VIII.2005, SR, 1 es. (CSR).

COROTIPO: Europeo (EUR).

Riolus nitens (P.W.J. Müller, 1817)

RePerti (1): Canneto (PI), t. Ritasso, m 100, 15.VI.2016, SR, 1 es. (CSR).

COROTIPO: Europeo-Mediterraneo (EUM).

Stenelmis consobrina consobrina Dufour, 1835

Reperti (2): Casino di Terra (PI), f. Cecina, 8.VIII.1996, FT, 1 es. (CFT); Pomarance (PI), f. Cecina, m 87, 4.VIII.2005, FT, 1 es. (CFT), SR, 3 es. (CSR).

Corotipo: Centroasiatico-Europeo-Mediterraneo (CEM).

\section{Dryopidae}

Dryops algiricus (Lucas, 1846)

RePerti (5): Casino di Terra (PI), t. Sterza, m 41, 21.VIII.2001, SR, 1 es. (CSR); Gello (PI), laghetto presso podere Sant'Alessandro, m 165, 16.V.2017, AM, FT, SR, 1 es. (CAM), 1 es. (CFT), 1 es. (CSR); Gello (PI), stagno presso il cimitero, $\mathrm{m}$ 143, 16.V.2017, AM, FT, SR, 3 es. (CAM), 4 es. (CFT), 8 es. (CSR); Riparbella (PI), t. Lopia presso podere Santa Margherita, m 60-63, 16.V.2017, AM, FT, SR, 9 es. (CAM), 5 es. (CFT), 2 es. (CSR); Volterra (PI), confluenza del f. Cecina con rio del Gagno, $\mathrm{m} 70$ (Mascagni et al., 1997).

COROTIPO: Mediterraneo (MED).

\section{Dryops lutulentus (Erichson, 1847)}

RePerti (10): Canneto (PI), t. Ritasso, m 100, 15.VI.2016, SR \& FT, 3 es. (CSR), 1 es. (CFT); Casino di Terra (PI), t. Sterza, m 41, 21.VIII.2001, 1 ex; ibidem, 4.VIII.2005, FT, 2 es. (CFT), SR, 2 es. (CSR); Casole d'Elsa (SI), t. Sellate, m 250 (Mascagni et al., 1997); Castelnuovo di Val di Cecina (PI), t. Pavone, m 250 (Mascagni et al., 1997); Castelnuovo di Val di Cecina (PI), t. Pavone, m 330, 27.VIII.2003, SR, 2 es. (CSR); Pomarance (PI), f.
Cecina, m 87, 4.VIII.2005, SR, 1 es. (CSR); Radicondoli (SI), Bagni delle Galleraie, f. Cecina, m 340 (Mascagni, 2005b); Radicondoli (SI), f. Cecina, m 222 (Mascagni, 2005b); Saline di Volterra (PI), f. Cecina, m 70 (Mascagni et al., 1997).

Corotipo: Centroasiatico-Europeo-Mediterraneo (CEM).

Dryops subincanus (Kuwert, 1890)

Reperti (10): Casino di Terra (PI), f. Cecina, 7.VIII.1996, FT, 13 es. (CFT); Casino di Terra (PI), t. Sterza, m 41, 21.VIII.2001, SR, 1 es. (CSR); Monteguidi (SI), f. Cecina, m 200 (Mascagni et al., 1997); Pomarance (PI), f. Cecina, m 83, 17.IX.2020, AM, SR, FT, 2 es. (CAM), 5 es. (CFT), 3 es. (CSR); Pomarance (PI), f. Cecina, m 87, 4.VIII.2005, FT, 5 es. (CFT), SR, 1 es. (CSR); Pomarance (PI), t. Trossa, m 200 (Mascagni et al., 1997); Ponteginori (PI), t. Trossa, 9.VIII.1996, FT, 10 es. (CFT); Ponteginori (PI), t. Trossa, m 49, 8.VI.2014, SR, 2 es. (CSR); Saline di Volterra (PI), f. Cecina, m 70 (Mascagni et al., 1997); Volterra (PI), f. Cecina, m 70 (Mascagni et al., 1997). COROTIPO: Europeo-Mediterraneo (EUM).

Dryops sulcipennis (A. Costa, 1883)

Reperti (1): Casino di Terra (PI), f. Cecina, 7.VIII.1996, FT, 1 es. (CFT).

Corotipo: Mediterraneo (MED).

Pomatinus substriatus (P.W.J. Müller, 1806)

Reperti (10): Canneto (PI), fosso di Malentrata, m 140, 15.VI.2016, SR, 2 es. (CSR); Canneto (PI), t. Ritasso, m 100, 1.VI.2016, SR, 4 es. (CSR); Canneto (PI), t. Ritasso, m 164, 29.V.2018, SR, 1 es. (CSR); Castelnuovo di Val di Cecina (PI), t. Pavone, $\mathrm{m}$ 330, 27.VIII.2003, SR, 1 es. (CSR); Pomarance (PI), f. Cecina, m 83, 17.IX.2020, AM, SR, FT, 6 es. (CAM), 3 es. (CFT), 1 es. (CSR); Pomarance (PI), t. Trossa, m 200 (Mascagni et al., 1997); Ponte di Cecina (SI), f. Cecina, m 259, 26.VII.2007, SR, 2 es. (CSR); Radicondoli (SI), Bagni delle Galleraie, f. Cecina, m 340 (Mascagni, 2005b); Riparbella (PI), t. Le Botra presso Mulino del Frullino, m 66-70, 16.V.2017, AM, FT, SR, 3 es. (CAM), 1 es. (CFT), 1 es. (CSR); Riparbella (PI), t. Lopia presso podere Santa Margherita, m 60-63, 16.V.2017, AM, FT, SR, 3 es. (CAM), 2 es. (CFT), 2 es. (CSR).

CoROTIPO: Turanico-Europeo-Mediterraneo (TEM). 


\section{Limnichidae}

Pelochares versicolor (Waltl, 1838)

REPERTI (2): Gello (PI), laghetto presso podere Sant'Alessandro, m 165, 16.V.2017, AM, 1 es. (CAM); Volterra (PI), confluenza del f. Cecina con rio del Gagno, m 70 (Mascagni et al., 1997).

COROTIPO: Europeo-Mediterraneo (EUM).

\section{Heteroceridae}

Augyles (Augyles) flavidus (P. Rossi, 1794)

RePerti (1): Volterra (PI), confluenza del f. Cecina con rio del Gagno, m 70 (Mascagni, 2005b).

Corotipo: Centroasiatico-Europeo-Mediterraneo (CEM).

Augyles (Augyles) marmota (Kiesenwetter, 1850) Reperti (2): Casole d'Elsa (SI), t. Sellate, m 225 (Mascagni, 2005b); Guardistallo (PI), t. Sterza, m 45 presso podere Aizzi (Mascagni, 2005b).

Corotipo: Centroasiatico-Europeo-Mediterraneo (CEM).

Heterocerus fenestratus (Thunberg, 1784)

REPERTI (7): Casole d'Elsa (SI), t. Sellate, m 230 (Mascagni, 2005b); Gello (PI), laghetto presso podere Sant'Alessandro, m 165, 16.V.2017, AM, 1 es. (CAM); Guardistallo (PI), Pian del Molino, f. Cecina, m 40 (Mascagni, 2005b); Guardistallo (PI), t. Sterza, m 45-50 presso podere Aizzi (Mascagni, 2005b); Pomarance (PI), f. Cecina, m 80 (Mascagni, 2005b); Riparbella (PI), t. Lopia presso podere Santa Margherita, m 60-63, 16.V.2017, AM, 1 es. (CAM); Volterra (PI), confluenza del f. Cecina con rio del Gagno, $\mathrm{m} 70$ (Mascagni, 2005b).

COROTIPO: Olartico (OLA).

Heterocerus holosericeus Rosenhauer, 1856

RePerti (5): Guardistallo (PI), Pian del Molino, f. Cecina, m 45 (Mascagni, 2005b); Guardistallo (PI), t. Sterza, m 50 presso podere Aizzi (Mascagni, 2005b); Ponteginori (PI), t. Trossa, m 49, 8.VI.2014, SR, 1 es. (CSR); Ponteginori (PI), t. Trossa, m 50 (Mascagni, 2005b); Volterra (PI), confluenza del f. Cecina con rio del Gagno, m 70 (Mascagni, 2005b).

COROTIPO: Indiano-Mediterraneo (INM).

\section{Chrysomelidae}

Donacia cinerea Herbst, 1784

RePerti (1): Monteguidi (SI), f. Cecina, m 200, 6.VI.2001, SR, 2 es. (CSR).

CoRotiPo: Sibirico-Europeo (SIE).

\section{CONCLUSIONI}

I taxa (specie e sottospecie) elencati per il bacino idrografico del fiume Cecina sono complessivamente 120 suddivisi in 14 famiglie: Gyrinidae (6), Haliplidae (5), Noteridae (1), Dytiscidae (31), Helophoridae (8), Georissidae (2), Hydrochidae (2), Hydrophilidae (20), Hydraenidae (26), Elmidae (8), Dryopidae (5), Limnichidae (1), Heteroceridae (4), Chrysomelidae (1).

Complessivamente sono stati elencati 589 reperti, di cui 220 tratti da dati bibliografici e 369 inediti; il maggior numero di reperti inediti rispetto a quelli editi si riscontra soprattutto negli Helophoridae (43 contro 9), negli Hydrophilidae (89 contro 53), negli Hydraenidae (92 contro 37); addirittura per gli Elmidae, a fronte di un unico reperto edito, ne abbiamo registrati altri 10 inediti; evidentemente si tratta di famiglie che nel passato, in questa zona, non sono state oggetto di particolari ricerche.

Per la zona indagata risultavano già noti 89 taxa, rilevando adesso la presenza di altre 31 specie: 2 Haliplidae (Haliplus obliquus, H. variegatus), 6 Dytiscidae (Agabus brunneus, Rhantus suturalis, Hydaticus leander, Hydroporus analis, Stictotarsus duodecimpustulatus, Laccophilus poecilus), 4 Helophoridae (Helophorus alternans, H. aquaticus, H. flavipes, H. griseus), 3 Hydrophilidae (Enochrus testaceus, Paracymus aeneus, Coelostoma orbiculare), 7 Hydraenidae (Hydraena andreinii, Limnebius atomus, L. mucronatus, L. oblongus, Ochthebius halberri, O. lividipennis, O. pusillus), 7 Elmidae (Elmis aenea, Esolus berthelemyi, Limnius intermedius, $L$. opacus, Riolus cupreus, $R$. nitens, Stenelmis consobrina), 1 Dryopidae (Dryops sulcipennis), 1 Chrysomelidae (Donacia cinerea); la biodiversità precedentemente nota è risultata pertanto incrementata del 34,83\%.

Per l'analisi corologica abbiamo suddiviso i corotipi fondamentali dei suddetti 120 taxa in gruppi di più ampio significato zoogeografico, ottenendo così i valori riportati nella Tab. 1. Molto elevata risulta la percentuale $(57,51 \%)$ delle specie appartenenti al 
gruppo ad ampia distribuzione olartica, dovuta alla notevole presenza di specie euriecie che, per la loro elevata valenza ecologica, sono caratterizzate da una larga diffusione. Si rilevano inoltre considerevoli percentuali anche per le specie appartenenti ai corotipi Europeo-Mediterraneo (23,35\%), Sud Europeo $(18,34 \%)$ e Mediterraneo (10,84\%) Nei bacini del fiume Sieve (Cianferoni et al., 2014) le percentuali di queste categorie corotipiche risultano invece inferiori essendo rispettivamente del $14,65 \%, 19,75 \%$ e $5,10 \%$ e quelle del torrente Pesa (Rocchi \& Terzani, 2016) del $16,66 \%, 16,66 \%$ e $9,26 \%$; evidentemente ciò è dovuto alla minore continentalità del bacino del fiume Cecina (derivante dalla maggiore vicinanza al litorale marino) e dall'assenza di aree tipicamente montane e submontane. La percentuale $(10 \%)$ dei taxa endemici del bacino del fiume Cecina (tutti appartenenti al corotipo Sud Europeo) è quasi uguale a quella del bacino del fiume Sieve $(10,13 \%)$ e del bacino del torrente Pesa $(9,26 \%)$.
Abbiamo inoltre confrontato i dati faunistici del bacino del fiume Cecina con quelli dei due bacini sopra mezionati, sia a livello di similarità che come numero di taxa di ciascuna famiglia; per quanto riguarda la similarità abbiamo calcolato il quoziente di Sørensen [QS $=2 c /(a+b)]$, dove $c=$ numero di specie in comune, $a+b=$ numero di specie presenti nei due bacini, ottenendo i seguenti quozienti: Cecina $/$ Sieve $=0,62 ;$ Sieve $/$ Pesa $=0,70 ;$ Cecina $/$ Pesa $=$ 0,73 ; la maggiore similarità risulta pertanto fra il bacino del fiume Cecina e quello del torrente Pesa, mentre è minore fra il bacino del fiume Cecina e quello del fiume Sieve, a conferma di quanto riscontrato anche a livello corologico per la differente posizione geografica e la diversa situazione altimetrica di questi tre bacini (quote delle nostre ricerche: $\mathrm{m}$ 78-1300 per il bacino del fiume Sieve, m 35-589 per quello del torrente Pesa e m 40-350 per quello del fiume Cecina).

Per il confronto del numero di taxa di ciascuna

Tab. 1. Quadro corologico.

\begin{tabular}{|c|c|c|c|c|c|}
\hline Gruppi di corotipi & $\begin{array}{l}\text { Numero } \\
\text { di specie }\end{array}$ & $\begin{array}{l}\text { Valore } \\
\%\end{array}$ & $\begin{array}{l}\text { Sigla } \\
\text { corotipi }\end{array}$ & $\begin{array}{l}\text { Numero } \\
\text { di specie }\end{array}$ & $\begin{array}{l}\text { Valore } \\
\%\end{array}$ \\
\hline Subcosmopoliti & 1 & 0,83 & $\mathrm{SCO}$ & 1 & 0,83 \\
\hline \multirow{10}{*}{ Ampia distribuzione olartica } & \multirow{10}{*}{69} & \multirow{10}{*}{57,51} & OLA & 1 & 0,83 \\
\hline & & & PAL & 6 & 5,00 \\
\hline & & & WPA & 2 & 1,66 \\
\hline & & & ASE & 4 & 3,34 \\
\hline & & & SIE & 8 & 6,67 \\
\hline & & & CEM & 8 & 6,67 \\
\hline & & & $\mathrm{CAE}$ & 1 & 0,83 \\
\hline & & & TEM & 9 & 7,50 \\
\hline & & & TUE & 2 & 1,66 \\
\hline & & & EUM & 28 & 23,35 \\
\hline \multirow[t]{3}{*}{ Ampia distribuzione in Europa } & \multirow{3}{*}{31} & \multirow{3}{*}{25,84} & EUR & 8 & 6,67 \\
\hline & & & $\mathrm{CEU}$ & 1 & 0,83 \\
\hline & & & SEU & 22 & 18,34 \\
\hline \multirow[t]{3}{*}{ Ampia distribuzione nel Mediterraneo } & \multirow{3}{*}{17} & \multirow{3}{*}{14,16} & MED & 13 & 10,84 \\
\hline & & & WME & 2 & 1,66 \\
\hline & & & EME & 2 & 1,66 \\
\hline \multirow{2}{*}{$\begin{array}{l}\text { Ampia distribuzione paleotropicale estesa } \\
\text { all'area mediterranea }\end{array}$} & \multirow{2}{*}{2} & \multirow{2}{*}{1,66} & AFM & 1 & 0,83 \\
\hline & & & INM & 1 & 0,83 \\
\hline Totali & 120 & 100,00 & & 120 & 100,00 \\
\hline \multicolumn{4}{|l|}{ di cui specie endemiche } & 12 & 10,00 \\
\hline
\end{tabular}


famiglia abbiamo riepilogato i dati nella Tab. 2, rilevando che per i bacini del torrente Pesa e del fiume Cecina, rispetto a quelli del fiume Sieve, risulta particolarmente evidente il minor numero di specie dei Dytiscidae, degli Hydraenidae e degli Elmidae; ciò è da mettere in relazione non soltanto alle differenze ambientali sopra menzionate ma anche alla diversa ampiezza dei relativi bacini (Sieve $\mathrm{km}^{2} 843$, Pesa $\mathrm{km}^{2}$ 334, Cecina $\mathrm{km}^{2} 765$ ).

Fra le specie molto comuni del bacino del fiume Cecina possiamo annoverare quelle che risultano segnalate con 15 o più reperti e cioè: Helophorus brevipalpis, H. obscurus, Laccobius albescens, L. neapolitanus, Hydraena subimpressa, Ochthebius crenulatus, tutte caratterizzate da un'elevata capacità di adattamento ecologico e infatti presenti anche in entrambi gli altri due bacini oggetto di questo confronto.
Molte di più sono invece le specie che si sono rivelate rare; possiamo considerare tali tutte quelle dove risulta segnalato un solo reperto (spesso alquanto datato) e in particolare alcune notoriamente sporadiche in quanto biologicamente ed ecologicamente specializzate a vivere in ambienti molto particolari, come per esempio Gyrinus paykulli (biotopi di acque fredde con canneti), Georissus crenulatus e G. costatus (nella sabbia sul bordo delle rive soprattutto di acque correnti), Paracymus aeneus (in acque lentiche semisalate), Cercyon granarius (al margine di acque stagnanti), Hydraena solarii e Riolus nitens (in ambienti reofili), Donacia cinerea (sulla vegetazione ripariale, soprattutto su foglie di Thypha). Queste pur brevi indicazioni ecologiche si riferiscono a biotopi con un buon livello di naturalità, meritevoli pertanto di grande attenzione conservazionistica.

Tab. 2. Confronto fra il numero di taxa (specie e sottospecie) dei bacini idrografici del fiume Sieve, del torrente Pesa e del fiume Cecina.

\begin{tabular}{|c|c|c|c|}
\hline Famiglie & $\begin{array}{c}\text { Fiume Sieve } \\
\text { (bacino } \mathrm{km}^{2} 843 \text { ) }\end{array}$ & $\begin{array}{c}\text { Torrente Pesa } \\
\text { (bacino } \mathrm{km}^{2} 334 \text { ) }\end{array}$ & $\begin{array}{c}\text { Fiume Cecina } \\
\text { (bacino } \mathrm{km}^{2} 765 \text { ) }\end{array}$ \\
\hline Sphaeriusidae & 1 & - & - \\
\hline Gyrinidae & 4 & 5 & 6 \\
\hline Haliplidae & 6 & 6 & 5 \\
\hline Noteridae & 1 & 1 & 1 \\
\hline Dytiscidae & 48 & 29 & 31 \\
\hline Helophoridae & 11 & 5 & 8 \\
\hline Georissidae & 1 & - & 2 \\
\hline Hydrochidae & 3 & 2 & 2 \\
\hline Hydrophilidae & 16 & 17 & 20 \\
\hline Hydraenidae & 34 & 22 & 26 \\
\hline Scirtidae & 4 & - & - \\
\hline Elmidae & 15 & 8 & 8 \\
\hline Dryopidae & 5 & 4 & 5 \\
\hline Limnichidae & 2 & 3 & 1 \\
\hline Heteroceridae & 5 & 4 & 4 \\
\hline Chrysomelidae & 1 & 1 & 1 \\
\hline Curculionidae & 1 & 1 & - \\
\hline Totali & 158 & 108 & 120 \\
\hline
\end{tabular}




\section{RINGRAZIAMENTI}

Si ringraziano gli amici e colleghi che hanno permesso di esaminare alcuni degli esemplari citati in questo contributo: Luca Bartolozzi (Firenze); Simone
Cianfanelli (Firenze); Fabio Cianferoni (Firenze); Leonardo Forbicioni (Portoferraio, Livorno); Rossano Papi (Castelfranco di Sopra, Arezzo); Lucia Pizzocaro (Vezza d'Oglio, Brescia); Roberto Poggi (Genova).

\section{BIBLIOGRAFIA}

ANGelnN F., 1984 - Catalogo topografico dei Coleoptera Haliplidae, Hygrobiidae, Dytiscidae e Gyrinidae. Memorie della Società entomologica italiana, 61A (1982): 45-126.

ANGus R.B., Toledo M, 2010 - A new, chromosomally distinct italian Helophorus F. similar to H. aequalis Thomson, and notes on some other species in Italy (Coleoptera: Helophoridae). Koleopterologische Rundschau, 80: 103-111.

Audisio P., De Biase A., 2005 - Insecta Coleoptera Hydraenidae, pp. 169-170. In: RufFo S., Stoch F. (eds.), Checklist e distribuzione della fauna italiana. Memorie del Museo civico di Storia naturale di Verona, 2. serie, Sezione Scienze della Vita, 16, 307 pp., più CD ROM.

ChIESA A., 1959 - Hydrophilidae Europae. Coleoptera Palpicornia. Tabelle di determinazione. Arnaldo Forni Editore, Bologna, $199 \mathrm{pp}$.

Cianferoni F., Rocchi S., Terzani F., MASCAgni A., 2014 - Coleotterofauna acquatica e semiacquatica del bacino idrografico del Fiume Sieve (Toscana) (Insecta Coleoptera). Bollettino della Società entomologica italiana, 146 (2): 51-81.

FERY H., 1992 - Revision der «minutissimus-Gruppe» der Gattung Bidessus Sharp (Coleoptera: Dytiscidae). Entomologica Basiliensia, 14 (1991): 57-91.

Guerrini M., Masetti M., Politi P.M., Tomei P.E., ZufFi M.A.L. (a cura di). 2008 - Approfondimenti conoscitivi su alcune aree d'interesse ambientale del Piano Territoriale di Coordinamento. Relazione finale, luglio 2008. Convenzione fra la Provincia di Pisa e il Dipartimento di Biologia dell’Università di Pisa, pp. 80.

HÁJEK J., 2017 - Family Noteridae C.G. Thomson, 1860 (pp. 844-845). In: LöBL I., LöBL D. (eds.), Catalogue of Palaearctic Coleoptera. Archostemata, Myxophaga, Adephaga. Revised and Updated Edition, Volume 1. Brill, Leiden/Boston, 1443 pp.

HÁJeK J., FerY H., 2019 - Catalogue of Palaearctic Gyrinidae (Coleoptera). Internet Version 1.01.2019 (http://www.waterbeetles.eu).

Hernando C., Ribera I., 2016 - Family Limnichidae Erichson, 1846 (pp. 607-610). In: LöBL I., LöBL D. (eds.), Catalogue of Palaearctic Coleoptera. Revised and Updated Edition. Volume 3. Scirtoidea - Byrrhoidea. Brill, Leiden/Boston, 983 pp.

JÄCH M.A., 1991 - Revision of the Palearctic species of the genus Ochthebius VII. The foveolatus group (Coleoptera: Hydraenidae). Koleopterologische Rundschau, 61: 61-94.

JÄCH M.A., 1998 - Annotated check list of aquatic and riparian/littoral beetle families of the world (Coleoptera) (pp. 25-42). In: JÄCH M.A., JI L. (eds.). Water Beetles of China. Vol. II. Zoologisch-Botanische Gesellschaft and Wiener Coleopterologenverein, $371 \mathrm{pp}$.

JäCH M.A., KoDADA J., 2016 - Elmidae (pp. 591-603). In: LöBL I., LÖBL D. (eds.). Catalogue of Palaearctic Coleoptera. Revised and Updated Edition. Volume 3. Scirtoidea - Byrrhoidea. Brill, Leiden/Boston, 983 pp.

Jä́h M.A., SKale A., 2015 - Hydraenidae (pp. 130-162). In: LöBL I., LöBL D. (eds.). Catalogue of Palaearctic Coleoptera. Revised and Updated Edition. Volume 2/1. Hydrophiloidea - Staphylinoidea. Brill, Leiden/Boston, 1702 pp.

KodaDa J., JÄCH M.A., 2016 - Dryopidae (pp. 603-607). In: LöBL I., LöBL D. (eds.). Catalogue of Palaearctic Coleoptera. Revised and Updated Edition. Volume 3. Scirtoidea - Byrrhoidea. Brill, Leiden/Boston, 983 pp.

MASCAGNI A., 2005a - Insecta Coleoptera Georissidae (pp. 171-172). In: RufFo S., Stoch F. (eds.), Checklist e distribuzione della fauna italiana. Memorie del Museo civico di Storia Naturale di Verona, 2. serie, Sezione Scienze della Vita, 16, 307 pp., più CD ROM.

Mascagni A., 2005b - Insecta Coleoptera Dryopoidea (pp.199-200). In: RufFo S., Stoch F. (eds.), Checklist e distribuzione della fauna italiana. Memorie del Museo civico di Storia naturale di Verona, 2. serie, Sezione Scienze della Vita, 16, 307 pp., più CD ROM.

MascagnI A., 2016 - Family Heteroceridae (pp. 610-616). In: LöBL J., LöBL D. (eds.), Catalogue of Palaearctic Coleoptera. Scarabaeoidea - Scirtoidea - Dascilloidea - Buprestoidea - Byrrhoidea. Revised and Updated Edition, Volume 3. Brill, Leiden/Boston, pp. 983.

Mascagni A., CAlAmandRei S., 1996 - Primo contributo alla conoscenza degli Hydraenidae, Spercheidae, Hydrochidae della Toscana (Coleoptera, Hydrophiloidea). Atti del Museo civico di storia naturale di Grosseto, 15 (1993): 9-22.

Mascagni A., Rocchi S., Terzani F., Calamandrei S., 1997 - Contributo alla conoscenza dei Coleotteri degli ambienti acquatici 
della Toscana. I. Psephenidae, Limnichidae, Driopidae, Elmidae (Coleoptera, Dryopoidea). Quaderni del Museo di Storia Naturale di Livorno, 14 (1995-1996): 47-78.

Minelli A., Ruffo S., Stoch F., 2005 - L’endemismo in Italia (pp. 29-32). In: Ruffo S., Stoch F. (eds.). Checklist e distribuzione della fauna italiana. Memorie del Museo civico di Storia Naturale di Verona, 2. serie, Sezione Scienze della Vita, 16, 307 pp., più CD ROM.

Nilsson A.N., HÁJeK J., 2020 - A World Catalogue of the Family Dytiscidae, or the Diving Beetles (Coleoptera, Adephaga). Internet Version 1.01.2020 (http://www.waterbeetles.eu).

PrZewoźNy M., 2020 - Catalogue of Palearctic Hydrophiloidea (Coleoptera). Internet version 1.01.2020 (http://www.waterbeetles.eu).

Regione Toscana, 2005 - Piano di tutela delle acque della Toscana. Volume 5. Bacino Toscana costa. Edizioni Regione Toscana, Firenze, $309 \mathrm{pp}$.

Rocchi S., 2005a - Insecta Coleoptera Hydroadephaga, pp. 165-166. In: RufFo S., STOch F. (eds.), Checklist e distribuzione della fauna italiana. Memorie del Museo civico di Storia naturale di Verona, 2. serie, Sezione Scienze della Vita, 16, 307 pp., più CD ROM.

Rocchi S., 2005b - Insecta Coleoptera Hydrophiloidea, pp. 167-168. In: RuFFo S., STOch F. (eds.), Checklist e distribuzione della fauna italiana. Memorie del Museo civico di Storia naturale di Verona, 2. serie, Sezione Scienze della Vita, 16, 307 pp., più CD ROM.

Rocchi S., Terzani F., 2016 - Coleotterofauna acquatica e semiacquatica del bacino idrografico del torrente Pesa (Toscana) (Insecta: Coleoptera). Onychium, 12: 83-103.

RocChI S., TerZANi F., 2018 - Contributo alla conoscenza dei Coleotteri degli ambienti acquatici della Toscana (Italia Centrale). VI. Aggiornamenti: Sphaeriusidae, Helophoridae, Georisidae, Hydrochidae, Spercheidae, Hydrophilidae (Coleoptera). Onychium, 14: 109-130.

Rocchi S., Terzani F., MASCAgni A., 1999 - Contributo alla conoscenza dei Coleotteri degli ambienti acquatici della Toscana II. Hydraenidae (Coleoptera, Hydrophiloidea). Quaderni del Museo di Storia Naturale di Livorno, 15 (1997-1998): 3982.

Rocchi S., Terzani F., Mascagni A., 2002 - Contributo alla conoscenza dei Coleotteri degli ambienti acquatici della Toscana (Italia). III. Helophoridae, Hydrochidae, Hydrophilidae, Spercheidae, Sphaeriusidae, Georissidae (Coleoptera). Quaderni del Museo di Storia Naturale di Livorno, 16 (2001-2002): 7-59.

SCHÖDL S., 1993 - Revision der Gattung Berosus Leach. 3. Teil: Die paläarktischen und orientalischen Arten der Untergattung Berosus s. str. (Coleoptera: Hydrophilidae). Koleopterologische Rundschau, 63: 189-233.

Silfverberg H., 2010 - Family Chrysomelidae Latreille, 1802. Subfamily Donaciinae Kirby, 1837 (pp. 354-358). In: LÖBL I., Smetana A. (eds.), Catalogue of Palaearctic Coleoptera. Volume 6. Chrysomeloidea. Apollo Books, Stenstrup, 924 pp.

Stoch F., Vigna Taglianti A., 2005 - I corotipi della fauna italiana (pp. 25-28). In: Ruffo S., Stoch F. (eds.). Checklist e distribuzione della fauna italiana. Memorie del Museo civico di Storia Naturale di Verona, 2. serie, Sezione Scienze della Vita, 16, 307 pp. più CD ROM.

Targioni Tozzetti A., 1879 - Catalogo della collezione di insetti italiani del R. Museo di Firenze. Coleotteri. Serie 2a. Tipografia Cenniniana, Firenze: 31-62.

Touring Club Italiano, 2006 - Atlante stradale d'Italia. Volume Centro. Touring Editore S.r.l., Milano, 180 pp.

Vigna Taglianti A., Audisio P.A., Belfiore C., Biondi M., Bologna M.A., Carpaneto, G.M., De Biase A., De Felici S., Piattella E., Racheli T., Zapparoli M., Zoia S., 1993 - Riflessioni di gruppo sui corotipi fondamentali della fauna Wpaleartica ed in particolare italiana. Biogeographia (n.s.), 16 (1992): 159-179.

Vigna Taglianti A., Audisio P.A., Biondi M., Bologna M.A., Carpaneto G.M., De Biase A., Fattorini S., Piattella E., Sindaco R., Venchi A., Zapparoli M., 1999 - A proposal for a chorotype classification of the Near East fauna, in the framework of the Western Palearctic region. Biogeographia (n.s.), 20: 31-59.

Vondel B.J. van, 2017 - Family Haliplidae Aubé, 1836 (pp. 838-843) - In: LöBL I., LöBL D. (eds.), Catalogue of Palaearctic Coleoptera. Archostemata, Myxophaga, Adephaga. Revised and Updated Edition, Volume 1. Brill, Leiden/Boston, 1443 pp.

VONDEL B.J. VAN, 2019 - Features of the metacoxal air-storage space as additional characters for reconstructing the phylogeny of Haliplidae (Coleoptera). Tijdschrift voor Entomologie, 162: 13-32. 